\title{
Traceable Multiauthority Attribute-Based Encryption with Outsourced Decryption and Hidden Policy for CIoT
}

\author{
Suhui Liu $\mathbb{D}^{1},{ }^{1}$ Jiguo Yu $\mathbb{D},{ }^{2,3,4}$ Chunqiang $\mathrm{Hu} \mathbb{D}^{5,6}$ and Mengmeng $\mathrm{Li}^{1}$ \\ ${ }^{1}$ School of Computer Science, Qufu Normal University, Rizhao, 276826 Shandong, China \\ ${ }^{2}$ School of Computer Science and Technology, Qilu University of Technology (Shandong Academy of Sciences), Jinan, \\ Shandong 250353, China \\ ${ }^{3}$ Shandong Computer Science Center (National Supercomputer Center in Jinan), Jinan, Shandong 250014, China \\ ${ }^{4}$ Shandong Laboratory of Computer Networks, Jinan 250014, China \\ ${ }^{5}$ School of Big Data and Software Engineering, Chongqing University, Chongqing 400044, China \\ ${ }^{6}$ Key Laboratory of Dependable Service Computing in Cyber Physical Society, Ministry of Education (Chongqing University), China
}

Correspondence should be addressed to Jiguo Yu; jiguoyu@sina.com and Chunqiang Hu; chu@cqu.edu.cn

Received 31 October 2020; Revised 1 January 2021; Accepted 7 April 2021; Published 21 April 2021

Academic Editor: Yingjie Wang

Copyright $\odot 2021$ Suhui Liu et al. This is an open access article distributed under the Creative Commons Attribution License, which permits unrestricted use, distribution, and reproduction in any medium, provided the original work is properly cited.

Cloud-assisted Internet of Things (IoT) significantly facilitate IoT devices to outsource their data for high efficient management. Unfortunately, some unsettled security issues dramatically impact the popularity of IoT, such as illegal access and key escrow problem. Traditional public-key encryption can be used to guarantees data confidentiality, while it cannot achieve efficient data sharing. The attribute-based encryption (ABE) is the most promising way to ensure data security and to realize one-to-many fine-grained data sharing simultaneously. However, it cannot be well applied in the cloud-assisted IoT due to the complexity of its decryption and the decryption key leakage problem. To prevent the abuse of decryption rights, we propose a multiauthority $\mathrm{ABE}$ scheme with white-box traceability in this paper. Moreover, our scheme greatly lightens the overhead on devices by outsourcing the most decryption work to the cloud server. Besides, fully hidden policy is implemented to protect the privacy of the access policy. Our scheme is proved to be selectively secure against replayable chosen ciphertext attack (RCCA) under the random oracle model. Some theory analysis and simulation are described in the end.

\section{Introduction}

In traditional public key encryption schemes, the encryptor encrypts the message with the public key of the decryptor; hence, only the decryptor who owns the corresponding decryption key can decrypt the data. In other words, this type of scheme relies on the public key certificate system which we all know is pretty difficult to manage. In 1984, Shamir first proposed the identity-based encryption (IBE) where the encryptor uses the identity of the decryptor as his/her public key [1]. In [2], Boneh et al. proposed an IBE using the elliptic curve pairing, which greatly promoted the development of this field. Although the IBE solves the public key management problem, it still cannot achieve one-to-many private data sharing. Unfortunately, this kind of application is extremely common in ubiquitous Internet of Things (IoT) scenarios.

To tackle this issue, Sahai et al. first proposed a fuzzy identity encryption scheme [3], which is later developed into the attribute-based encryption (ABE). There are two types of $\mathrm{ABE}$, the first one is named as ciphertext-policy attributebased encryption (CP_ABE) and the other one is key-policy attribute-based encryption (KP_ABE). CP_ABE was proposed by Waters, in which the encryptor needs to know nothing about who can decrypt the ciphertext exactly, and he/she just encrypts the message with a self-defined access policy [4]. Any decryptor can decrypt correctly as long as its attribute set meets the access policy in the ciphertext. In other words, in CP_ABE schemes, data owners own the right to design who can decrypt fully. 
IoT, which acts as the bridge between the physical world and the cyber world, enables the creation of a bunch of smart applications [5], such as smart city, smart industry, and smart health care system. Considering that most IoT devices are resource constrained and cannot handle the huge amount of data locally and efficiently, the cloud storage server is included in the IoT and forms a new paradigm, the cloudIoT, where the cloud or a resource-adequated server provides useful services like storage and computing. ABE schemes with a single attribute authority do not adequately address the needs of the ubiquitous IoT devices properly. In [6], Chase first proposed a multiauthority ABE scheme. However, Chase's scheme still requires the trusted central authority (CA), which can decrypt any ciphertext that it wants to decrypt. Later, Chase et al. improve their scheme by removing the CA and achieve a truly decentralized ABE scheme [7].

In [8], Lewko et al. proposed a distributed ABE scheme, which not only realizes multiauthority attribute-based encryption (MAABE) but also proves the system security with dual system encryption methodology. Unfortunately, the application of ABE in IoT still faces an important challenge: IoT devices with limited resources cannot afford the huge number of bilinear pairing operations in $\mathrm{ABE}$ schemes. Therefore, Green et al. proposed an outsourced ABE scheme which ensures the data security while minimizing the computational burden of equipments [9].

In this paper, a multiauthority attribute-based encryption scheme with white-box traceability and verifiable outsourced decryption was proposed for cloud IoT. Compared with the existing ABE schemes, our scheme has the following contributions:

(i) As there is a great quantity of attributes used in the decryption key generation, each attribute authority controlls a set of disjoint attributes independently in our scheme. The central authority is only responsible for generating the public parameters, and the right to decide who can decrypt is hold by the data owners directly

(ii) Our scheme uses the linear secret sharing schemes (LSSS) to allow any monotone access structures. More importantly, to protect the privacy of IoT users, our scheme realizes fully hidden access policy

(iii) Considering the needs of resource-constrained IoT devices, our scheme outsources most decryption works to the cloud by the verifiable outsourcing technology

(iv) Our scheme adopts the Boneh-Boyen short signature algorithm to implement the user traceability mechanism. In other words, we use a white-box trace algorithm to tackle the private key leaking issue

1.1. Paper Organizaiton. Section 2 summarizes many related works, and Section 3 introduces all preliminaries of our scheme including some complexity assumptions. The system model and security models are presented in Section 4. In Section 5 , we propose the concrete construction and a simple application of our scheme. Section 6 outlines the proof of indistinguishability, verifiability, fully hiding, and traceability of our scheme. We compare our scheme with some other schemes about the storage and computation costs in Section 7. Section 8 contains the conclusion.

\section{Related Work}

Many works have been proposed since Sahai et al. first proposed the attribute-based encryption [3]. ABE schemes can be classified into two categories generally: the key-policy attribute-based encryption (KP_ABE) and the ciphertextpolicy attribute-based encryption (CP_ABE) $[4,16]$. Because CP_ABE allows the data owner to decide the access policy, it has been treated as the most promising solution to solve the access control issue in the cloud storage. In ABE schemes, the key pair of data users is generated by attribute authorities (AAs). Thus, the security of ABE schemes is based on the trust of the attribute authorities. To tackle the huge amount of data users contained in IoT, multiauthority attributebased encryption (MA_ABE) was proposed, which can manage the huge amount of attributes in a more efficient way [6, 17-19], where each attribute authority controls an unique set of attributes independently. To achieve both the data confidentiality and the data authentication in the body area network, $\mathrm{Hu}$ et al. proposed a fuzzy attribute-based signcryption scheme [20].

Another characteristic of IoT is that most devices are resource-limited [21-23]. As we all know that the decryption overhead of $\mathrm{ABE}$ schemes rises along with the attribute number involved in the access policy. Obviously the expensive pairing computations are unacceptable for most IoT devices. Therefore, some ABE schemes using the proxy reencryption concept have been proposed [24-26]. In [9], Green et al. proposed an outsourced ABE scheme, which outsources most decryption overheads to a trusted third-party server, but outsourced ABE schemes all rely on a semitrusted server to semidecrypt that leads to a serious problem: how to ensure the semidecrypted data is correct and not altered. In [27], Lai et al. proposed a verifiable outsourced ABE while this scheme requires heavy costs for decryption. Recently, Li et al. improved an ABE scheme to achieve not only verifiable outsourced decryption but also lightweight user decryption [10], but all outsourced schemes mentioned above rely on a central authority to manage and generate user decryption key. In [11], Belguith et al. proposed an outsourced multiauthority attribute-based encryption scheme. In [28], Deng et al. proposed an efficient outsourced attribute-based signcryption scheme which also solves the user revocation problem.

In the cloud-assisted IoT environment, data owners store private data in the shared cloud. In most $\mathrm{ABE}$ schemes, the access policy is uploaded to the cloud server in plaintext along with the encrypted data. This may reveal private information of the encryptor and the decrypor. In [29], Nishide et al. proposed an $\mathrm{ABE}$ scheme with partially hided access policy, but this scheme has poor expressiveness.

When it comes to application in the real word, a common issue of $\mathrm{ABE}$ schemes needs to be considered: the leakage of 
decryption keys. In other words, how to trace/recover the global identity of the guilty user who leaks its secret key to a malicious or illegal user. There are two tracing approaches, white-box traceability and black-box traceability, that can be used to solve this issue. In [30], Hinek et al. used the BonehBoyen signature [31] to achieve the white-box traceability. Liu et al. proposed a white-box traceable $\mathrm{ABE}$ [32] and a black-box traceable $\mathrm{ABE}$ with highly expression [33]. In [12], Liu et al. proposed a traceable and revocable ABE scheme which is more practical for real application. In [34], $\mathrm{Yu}$ et al. proposed a traceable ABE scheme with white-box traceability to manage data stored in the cloud storage. In [13], an efficient large-universe MA_CP_ABE with whitebox traceability was proposed. While in [35], Qiao et al. proposed a traceable ABE scheme with black-box traceability for fog computing.

All traceable ABE schemes mentioned above have a shared issue: their decryption computation burden are intolerable for IoT devices. In [14], an ABE scheme with outsourced decryption designed for electronic health systems was proposed by Li et al. However, Li's scheme did not consider the privacy of access policies which might contain sensitive personal information of users. We compare our scheme with some existed ABE schemes in Table 1. In a word, our ABE scheme achieves selective replayable CCA security and provides multiple practical functions, such as fully hidden policy, outsourced decryption, and traceability.

\section{Preliminaries}

In this section, we provide all mathematical preliminaries needed for our scheme.

3.1. Bilinear Maps. Let $\mathbb{G}$ and $\mathbb{G}_{T}$ be two multiplicative cyclic groups of prime order $p$. Let $g$ be a generator of $\mathbb{G}$ and $e$ be a bilinearmap, e : $\mathbb{G} \times \mathbb{G} \rightarrow \mathbb{G}_{T}$, with the following three properties [15]:

(1) Bilinearity: for all $\mathrm{u}, v \in \mathbb{G}$ and $\mathrm{a}, b \in \mathbb{Z}_{p}$, we have $\mathrm{e}\left(u^{a}, v^{b}\right)=e(u, v)^{a b}$, where $\mathbb{Z}_{p}$ is the integers modulo $p$

(2) Nondegeneracy: $\mathrm{e}(g, g) \neq 1$, where 1 is the unit of $\mathbb{G}_{T}$

(3) Computability: there is a polynomial time algorithm to efficiently compute $\mathrm{e}(u, v)$ for any $\mathrm{u}, v \in \mathbb{G}$

We say $\mathbb{G}$ is a bilineargroup if the group operation in $\mathbb{G}$, and the bilinear map e : $\mathbb{G} \times \mathbb{G} \rightarrow \mathbb{G}_{T}$ is both efficiently computable. Notice that the map $e$ is symmetric since $\mathrm{e}\left(g^{a}, g^{b}\right)=e$ $(g, g)^{a b}=e\left(g^{b}, g^{a}\right)$.

\subsection{Access Structure}

Definition 1. (access structure). Let $P=\left\{P_{1}, \cdots P_{n}\right\}$ be a set of parties. A collection $A \subseteq 2^{\left\{P_{1}, \cdots P_{n}\right\}}$ is monotone if $\forall B, C$ : if $B \in \mathbb{A}$ and $B \subseteq C$, then $C \in \mathbb{A}$. An access structure is a collection $\mathbb{A}$ of nonempty subsets of $\left\{P_{1}, \cdots P_{n}\right\}$, such as $\mathbb{A} \subseteq$
$2^{\left\{P_{1}, \cdots P_{n}\right\}} \backslash \varnothing$. The sets in $\mathbb{A}$ are called authorized sets, and the sets not in $\mathbb{A}$ are called unauthorized sets [9].

\subsection{Linear Secret Sharing Schemes (LSSS)}

Definition 2. (linear secret sharing schemes (LSSS)). A secretsharing scheme $\prod$ over a set of parties $\mathbb{P}$ is called linear over $\mathbb{Z}_{p}$ if

(1) The shares of a secret $s \in \mathbb{Z}_{p}$ for each party form a vector over $\mathbb{Z}_{p}$

(2) There exists a matrix $\mathbb{M}$ with $l$ rows and $n$ columns called the share-generating matrix for $\prod$ and a function $\rho$ which maps each row of the matrix to an associated party. That is, for $i=1, . ., l$, the value $\rho(i)$ is the party associate with the row $i$. When we consider the column vector $\mathrm{v}=\left(s, r_{2}, \cdots, r_{n}\right)$ where $\mathrm{r}_{2}, \cdots, r_{n} \in \mathbb{Z}_{p}$ is randomly chosen, then $\mathbb{M} v$ is the vector of $l$ shares of the secret $s$ according to $\prod$. The share $(\mathbb{M} v)_{i}$ belongs to the party $\rho(i)$

According to [9], every linear secret-sharing scheme based on the above definition also enjoys the linear reconsruction property defined as follows: Let $\prod$ be an LSSS for the access structure $\mathbb{A}$. Let $S \in \mathbb{A}$ be any authorized set, and let $I \subset\{1$, $2, \cdots, l\}$ be defined as $\mathrm{I}=\{i: \rho(i) \in S\}$. Then, there exist constants $\left\{\omega_{i} \in \mathbb{Z}_{p}\right\}_{i \in I}$ such that if $\left\{\lambda_{i}\right\}$ are valid shares of any secret $s$ according to $\prod$, then $\sum_{\mathrm{i} \in \mathrm{I}} \omega_{i} \lambda_{i}=s$. It is shown in [9] that these constants $\left\{\omega_{i}\right\}$ can be found in polynomial time in the size of the share-generating matrix $\mathbb{M}$.

3.4. One-Way Anonymous Key Agreement. One-way anonymous key agreement [15] scheme can be used to guarantee anonymity of the access structure. This scheme only ensures the anonymity of one participant. Assume that there are two participants Alice $\left(\mathrm{ID}_{A}\right)$ and $\mathrm{Bob}\left(\mathrm{ID}_{B}\right)$ in this scheme. And the master secret of the key generation center (KGC) is $s$. When Alice wants to keep anonymity, the process is listed as follows:

(1) Alice calculates $Q_{B}=H\left(\operatorname{ID}_{B}\right)$. A random number $r_{a}$ $\in \mathbb{Z}_{p}^{*}$ is choosed to generate the pseudonym $\mathrm{P}_{A}=$ $Q_{A}^{r_{a}}$ and computes the session key $K_{A, B}=e\left(d_{A}, Q_{B}\right)^{r_{a}}$ $=e\left(Q_{A}, Q_{B}\right)^{s \cdot r_{a}}$. Finally, she sends her pseudonyms $\mathrm{P}_{A}$ to $\mathrm{Bob}$

(2) Bob uses his secret key $\mathrm{d}_{B}$ to calculate the session key $\mathrm{K}_{A, B}=e\left(P_{A}, d_{B}\right)=e\left(Q_{A}, Q_{B}\right)^{s \cdot r_{a}}$, where $\mathrm{d}_{i}=H\left(\mathrm{ID}_{i}\right)^{s}$ $\in \mathbb{G}$ is his private key for $i \in\{A, B\}$, and $H:\{0,1\}^{*}$ $\rightarrow \mathbb{G}$ is a strong collision-resistant hash function

\subsection{Complexity Assumptions}

Definition 3. Strong Diffie Hellman problem (q-SDH). Let $\mathbb{G}$ be a multiplicative cyclic group of order $p$ with a generator $g$. Given a random $x \in \mathbb{Z}_{p}^{*}$ and a $q+1$ tuple $\left(g, g^{x}, g^{x^{2}}, \cdots, g^{x^{q}}\right)$, 
TABle 1: Function comparison.

\begin{tabular}{lccccccc}
\hline Scheme & {$[10]$} & {$[11]$} & {$[12]$} & {$[13]$} & {$[14]$} & {$[15]$} & Our scheme \\
\hline Multiauthority & No & Yes & No & Yes & No & Yes & Yes \\
Access policy & AND gates & LSSS & LSSS & LSSS & LSSS & LSSS & LSSS \\
Fully hidden policy & No & No & No & No & No & Yes & Yes \\
Outdecryption & Yes & Yes & No & No & Yes & No & Yes \\
Security & RCCA & Selective RCPA & Selective CPA & Static security & Selective CPA & Selective CPA Selective RCCA \\
Traceability & No & No & Yes & Yes & Yes & No & Yes \\
\hline
\end{tabular}

the problem of computing a pair $\left(c, g^{1 / x+c}\right)$, where $c \in \mathbb{Z}_{p}^{*}$, is called the $q$-strong Diffie Hellman problem [13].

Definition 4. Computational Diffie Hellman problem (CDH). Let $\mathbb{G}$ be a multiplicative cyclic group of order $p$ with a generator $g$. Given two group elements $g^{\mathrm{a}}, \mathrm{g}^{\mathrm{b}} \in \mathbb{G}$ where $\mathrm{a}, \mathrm{b} \in$ $\mathbb{Z}_{\mathrm{p}}$ are two random integers. The problem of calculating $\mathrm{g}^{\mathrm{ab}}$ from $\mathrm{g}^{\mathrm{a}}$ and $\mathrm{g}^{\mathrm{b}}$ is called Computational Diffie Hellman problem [11].

Definition 5. Decisional Bilinear Diffie Hellman problem $(\mathrm{DBDH})$. Let $\mathbb{G}$ be a multiplicative cyclic group of order $p$ with a generator $g$. Given three group element $g^{a}, g^{b}$, and $g^{c} \in \mathbb{G}$ where $\mathrm{a}, \mathrm{b}$, and $c \in \mathbb{Z}_{\mathrm{p}}^{*}$ are three random integers. The problem of distinguishing tuples of the form $\left(g^{a}, g^{b}, g^{c}\right.$ ,$\left.e(g, g)^{a b c}\right)$ and $\left(g^{a}, g^{b}, g^{c}, e(g, g)^{z}\right)$ for some random integer $z$ is called the Decisional Bilinear Diffie Hellman problem [11].

\section{System Definition}

4.1. System Model. The system model of our scheme is illustrated in Figure 1, and the associated five entities are described as follows:

(1) Central Trusted Authority (CTA): the CTA is only used to generate the public parameter, and it cannot decrypted any data

(2) Attribute authorities (AAs): each AA controls a set of attributes. Multiple attribute authorities work together to generate the user's decryption key. Besides, attribute authorities can use a trace algorithm to recover the global identity of the guilty user who leaks its private decryption key

(3) Cloud storage service provider (CS): the CS is responsible to store the encrypted data. Moreover, CS performs the outsourcing decryption for users

(4) Data owner (DO): the owner of the data which is responsible to encrypt and upload the data to CS

(5) Data user (DU): the party who wants to access data

Table 2 summarizes notations used in our scheme. Assume that there are $n$ authorities in our scheme and each attribute is associated with an unique $\mathrm{AA}$, such that $\mathrm{S}_{\mathrm{A}, \mathrm{AA}_{i}}$ $\cap S_{{\mathrm{A}, \mathrm{AA}_{j}}}=\varnothing$ for $\forall i, j$ and $\bigcup S_{{\mathrm{A}, \mathrm{AA}_{j}}}=S_{A}$.

4.2. System Procedure. Our MAABE scheme with outsourced decryption and hidden policy contains the following five phases:

(1) System initialization: this phase includes two algorithms. Firstly, the CTA runs the $\operatorname{setup}(\lambda) \rightarrow \mathrm{PP}$ algorithm to generate the global parameters PP, where $\lambda$ is the security parameter. Then, each AA runs the $\operatorname{Setup}_{\text {auth }}(\mathrm{PP}) \rightarrow\left(\mathrm{sk}_{\mathrm{AAj}}, \mathrm{pk}_{\mathrm{AAj}}\right)$ algorithm to generate their own key pairs, which is consisted with a private key and a public key

(2) Encryption: the DO runs the $\operatorname{Encrypt}\left(\mathrm{PP},\left\{\mathrm{pk}_{\mathrm{AAj}}\right\}\right.$, MSG, $(\mathbb{M}, \rho)) \rightarrow$ CT algorithm to encrypt the message MSG, and then it uploads the ciphertext to the cloud server

(3) Keygeneration: this phase contains two algorithms. Firstly, each related AA runs the Keygen $\left(\mathrm{PP},\left\{s \mathrm{k}_{\mathrm{AAj}}\right.\right.$, $\left.\left.\mathrm{pk}_{\mathrm{AAj}}\right\}, \mathrm{GID}, \mathrm{S}_{\mathrm{GID}, \mathrm{j}}\right) \rightarrow \mathrm{sk}_{\mathrm{GID}, \mathrm{j}}$ algorithm independently to generate the decryption key for the DU with identity GID. Then, all results are sent to the user

To outsource the decryption work to the cloud, the user runs the $\operatorname{Keygen}_{\text {out }}\left(\mathrm{PP}, \mathrm{sk}_{\mathrm{GID}},(\mathbb{M}, \rho), \mathrm{CT}\right) \rightarrow \mathrm{ok}_{\mathrm{GID}}$ algorithm to generate its outsourced decryption key.

(4) Decryption: this phase is divided into two steps. Firstly, the CS runs the Decrypt ${ }_{\text {out }}\left(\mathrm{PP}, \operatorname{opk}_{\mathrm{GID}}\right.$, (M, $\rho), \mathrm{CT}) \rightarrow \mathrm{CT}^{\prime}$ algorithm to partially decrypt the ciphertext. The second step is performed by the user, who runs the $\operatorname{Decrypt}\left(C T^{\prime}\right.$, osk $\left._{\mathrm{GID}}\right) \rightarrow \mathrm{MSG}$ algorithm to get the plaintext

(5) Trace : to begin with, each $\mathrm{A} A_{j}$ verifies the format of the decryption key that needed to be traced, and then it runs the Trace $\left(P P, s k_{\mathrm{GID}},\left\{p k_{\mathrm{AA} j}\right\}\right) \rightarrow \mathrm{GID}$ algorithm to output the global identity (GID) of the guilty user

4.3. Security Models. We define four security models of our MAABE scheme in this section.

(1) Confidentiality: the confidentiality of data is the basic security requirement of a scheme, which is used to resist malicious adversaries to gain extral information from 


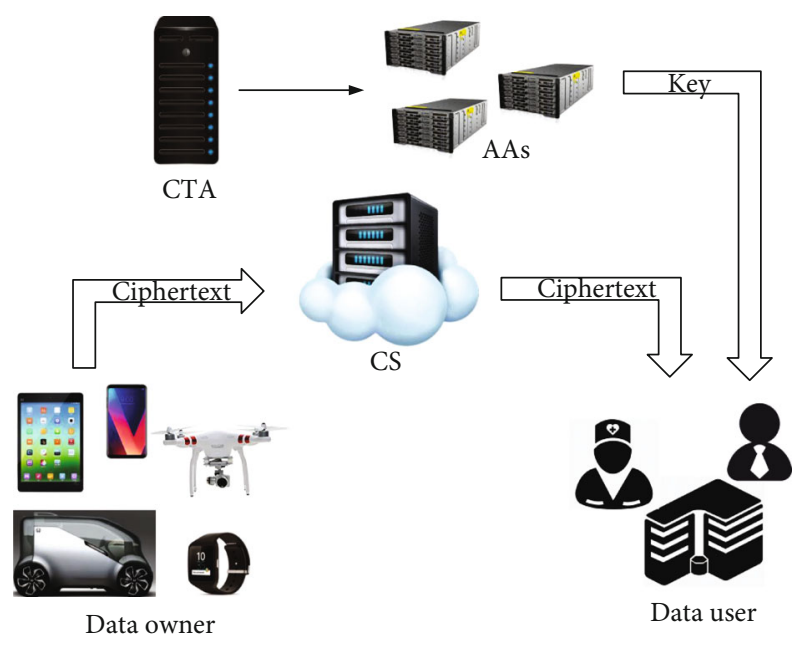

Figure 1: System model and procedure.

TABle 2: Notations.

\begin{tabular}{|c|c|}
\hline Notation & Meaning \\
\hline$S_{\mathrm{AA}}$ & The universe set of attribute authorities \\
\hline$S_{\mathrm{AA}}^{\prime}$ & The set of corrupted attribute authorities \\
\hline$S_{\mathrm{AA}, \mathrm{GID}}$ & The set of attribute authorities related to the user GID \\
\hline $\mathrm{S}_{\mathrm{A}}$ & The universe set of attributes \\
\hline $\mathrm{AA}_{j}$ & An attribute authority \\
\hline$S_{\mathrm{A}, \mathrm{A} A_{j}}$ & The attribute set controlled by $\mathrm{AA}_{j}$ \\
\hline GID & The global identity of a user \\
\hline$S_{\mathrm{GID}}$ & The attribute set related with user GID \\
\hline$P$ & The public parameter \\
\hline$s k_{\mathrm{AA}_{j}}$ & Secret key related to $\mathrm{AA}_{j}$ \\
\hline $\mathrm{p} k_{\mathrm{AA}_{j}}$ & Public key related to $\mathrm{AA}_{j}$ \\
\hline MSG & Message \\
\hline$(\mathbb{M}, \rho)$ & The LSSS access matrix and its row label function \\
\hline$\phi$ & Access control structure \\
\hline $\mathrm{S}_{\phi}$ & The attribute set related with $\phi$ \\
\hline $\mathrm{CT}$ & The ciphertext \\
\hline $\mathrm{sk}_{\mathrm{GID}}$ & The secret key of user GID \\
\hline $\mathrm{ok}_{\mathrm{GID}}$ & The outsourced decryption key ([opk, osk]) \\
\hline
\end{tabular}

the ciphertext. Our scheme adopts the replayable chosen-ciphertext security (RCCA) defined in [36] by Canetti et al. as this type of security is sufficient enough and not to be too strict. Two restrictions are followed in this experiment: all decryption key queries cannot satisfy the challenge access struction fixed in the initialization phase by the adversary. And the attribute authorities can only be corrupted statically be the adversary

The selective secure against chosen ciphertext attack of our scheme is achieved if no probabilistic polynomial time
(PPT) adversary can win the $\operatorname{Exp}^{\text {Conf }}$ security experiment described in Figure 2 between an adversary $\mathbb{A}$ and a challenger $\mathbb{C}$ with nonnegligible advantage.

(2) Verifiability: our scheme is verifiable if there is no PPT adversary that can win the Exp Verif security experiment described in Figure 3 between an adversary $\mathbb{A}$ and a challenger $\mathbb{C}$ with nonnegligible advantage.

(3) Fully hidden: in our scheme, the CS knows nothing about the access policy, and the user only knows if his/her attributes satisfy the access policy. Our scheme is an outsourced $\mathrm{ABE}$ with fully hidden policy if there is no PPT adversary that can win the Exp ${ }^{\text {Hide }}$ security experiment described in Figure 4 between an adversary $\mathbb{A}$ and a challenger $\mathbb{C}$ with nonnegligible advantage. The goal of the adversary is to recover the correct access policy without the required decryption key.

(4) Traceability: our scheme is a traceable ABE if there is no PPT adversary can win the Exp ${ }^{\text {Trace }}$ security experiment described in Figure 5 between an adversary $\mathbb{A}$ and a challenger $\mathbb{C}$ with nonnegligible advantage.

Definition 6. An outsourced ABE scheme is RCCA-secure against static corruption of the attribute authorities if $A d v_{\mathrm{A}}$ $\left[\operatorname{Exp}^{\operatorname{Conf}}\left(1^{\xi}\right)\right]$ is negligible for all PPT adversaries.

Definition 7. An outsourced ABE scheme is verifiable if $A d$ $v_{\mathrm{A}}\left[\operatorname{Exp}^{\operatorname{Verif}}\left(1^{\xi}\right)\right]$ is negligible for all PPT adversaries.

Definition 8. An outsourced ABE scheme achieves policy private if $A d v_{\AA}\left[\operatorname{Exp}^{\text {Priv }}\left(1^{\xi}\right)\right]$ is negligible for all PPT adversaries.

Definition 9. An outsourced ABE scheme is traceable if $A d$ $v_{\mathbb{A}}\left[\operatorname{Exp}^{\text {Trace }}\left(1^{\xi}\right)\right]$ is negligible for all PPT adversaries.

\section{Construction and Application}

The concrete construction of our MAABE scheme is presented in this section. Firstly, the CTA and all AA perform initialization and generate the PP and the public keys of AAs. Then, the DO can encrypt its data with an access structure. Before accessing the data, the DU needs to request its decryption key to the AAs. Next, the DU can access the data and decrypt successfully with the help of the cloud predecrypting for the DU first. Finally, the trace algorithm is used to reform the global identity of a guilty data user by the AAs. This section also contains a simple application in the end.

\subsection{Concrete Construction}

\subsubsection{Phase I: System Initialization}


Adversary

Challenger

Initialization -

Set up $\left\{\begin{array}{cc}\text { Setup auth }(\mathrm{PP}) \rightarrow\left(\mathrm{sk}_{\mathrm{AAj}}, \mathrm{pk}_{\mathrm{AAj}}\right) & \text { Corrupted attribute authorities: } \mathrm{S}_{\mathrm{AA}}^{\prime} \subset \mathrm{S}_{\mathrm{AA}} \\ \text { for corrupted attribute authorities } & \stackrel{\text { Public key set of non-corrupted AAs: }\left\{\mathrm{pk}_{\mathrm{AAj}}\right\}}{\longleftarrow}\end{array}\right.$ $\operatorname{Setup~}(\lambda) \rightarrow \mathrm{PP}$

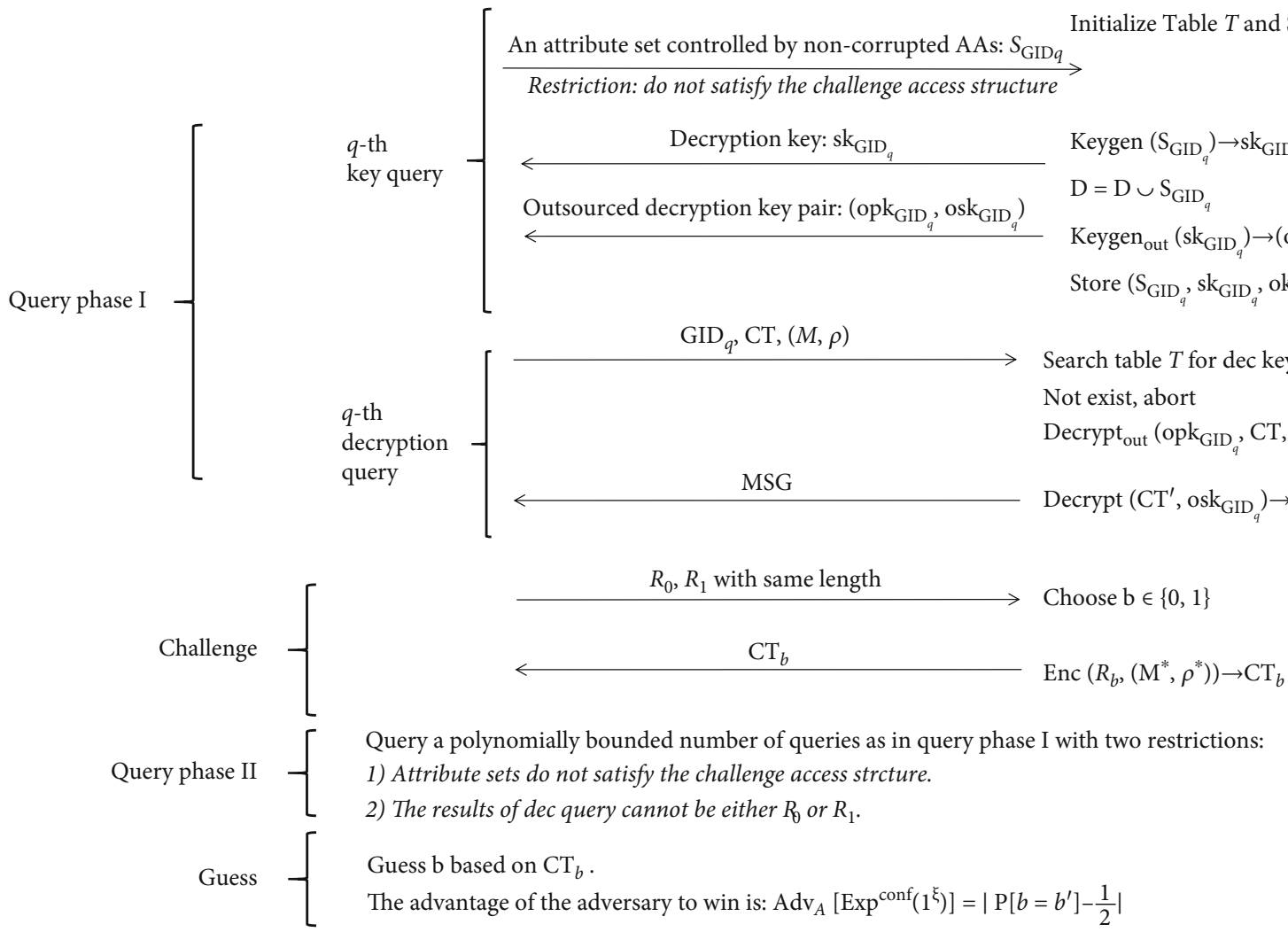

Figure 2: Confidentiality security model.

Adversary Challenger

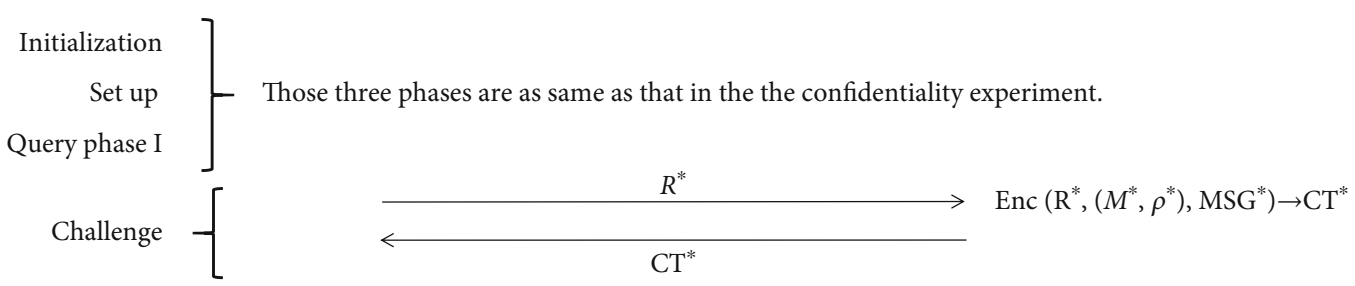

Query phase II Query a polynomially bounded number of queries as in query phase I with the restriction that attribute sets do not satisfy the challenge access strcture.

Assumption: The adversary already queried $\mathrm{S}_{\mathrm{GID}}^{*}$ in query phase I and stored the related key entry in table $T$.

Decrypt $_{\text {out }}\left(\right.$ opk $\left._{\text {GID }}^{*}, \mathrm{CT}^{*}\right) \rightarrow \mathrm{CT}^{\prime}$

Forge

Decrypt $\left(\mathrm{CT}^{\prime}\right.$, osk $\left.^{*}{ }_{\mathrm{GID}}\right) \rightarrow \mathrm{R}^{* \prime}$

The adversary wins this experiment if Decrypt $\left(\mathrm{R}^{* \prime}\right.$, osk $\left._{\mathrm{GID}}^{*}\right) \notin\left\{\mathrm{MSG}^{*}, \perp\right\}$

The advantage of the adversary to win is: $\operatorname{Adv}_{A}\left[\operatorname{Exp}^{\operatorname{Verif}}\left(1^{\xi}\right)\right]=\left|\operatorname{Pr}\left[\operatorname{Exp}^{\operatorname{Verif}}\left(1^{\xi}\right)\right]=1\right|$

FIGURE 3: Verifiability security model. 


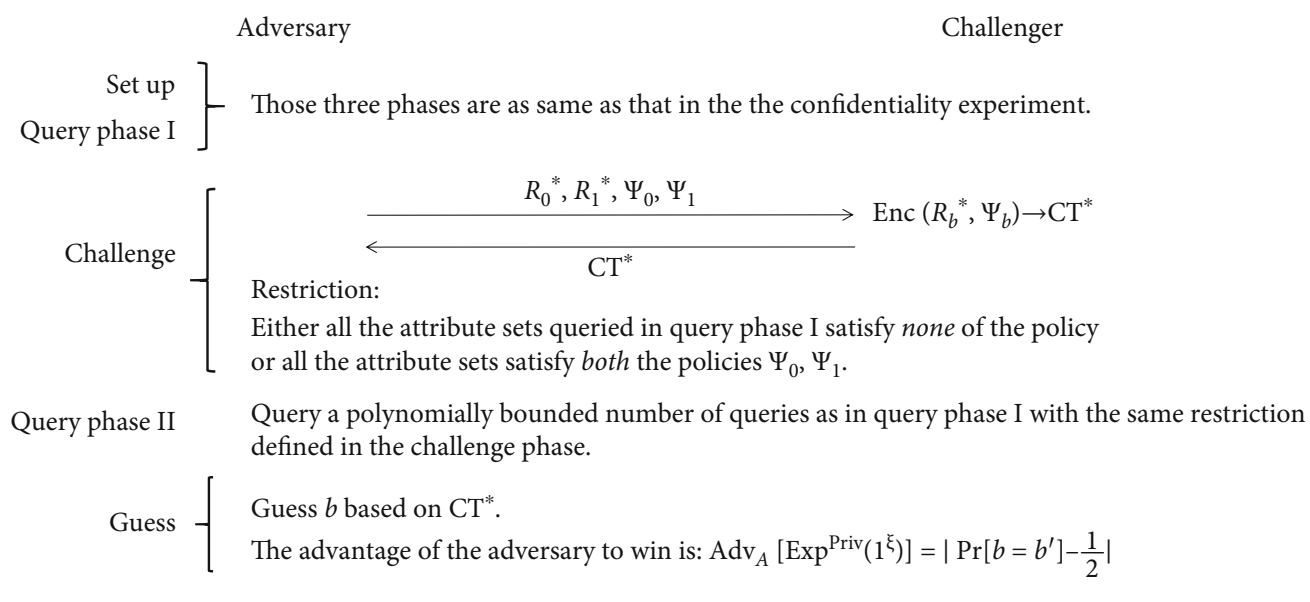

Figure 4: Fully hidden security model.

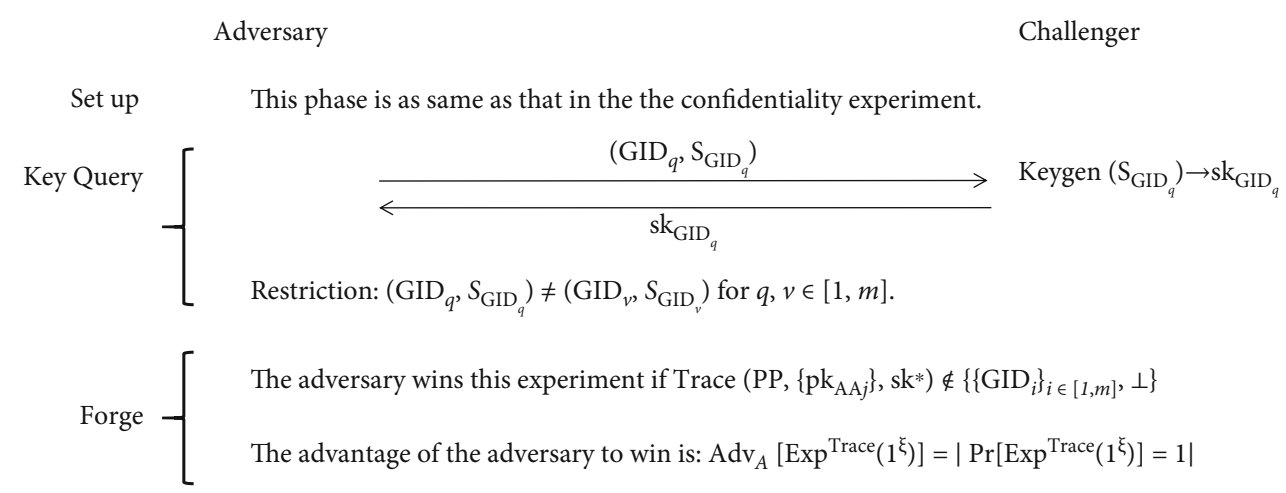

FIGURE 5: Traceability security model.

(1) System set-up : this step is performed by the CTA

It defines two multiplicative group $\mathbb{G}, \mathbb{G}_{T}$ of prime order $p$, and $g$ is a generator of $\mathbb{G}$.

It defines a symmetric bilinear map e : $\mathbb{G} \times \mathbb{G} \rightarrow \mathbb{G}_{T}$.

It defines three collusion resistant hash functions as follows: $\quad \mathrm{H}:\{0.1\}^{*} \rightarrow \mathbb{G}, H_{1}:\{0,1\}^{*} \rightarrow \mathbb{Z}_{p}^{*}, H_{2}:\{0,1\}^{*} \rightarrow$ $\{0,1\}^{k}$ where $k$ is the length of the symmetric key.

It defines a CPA-secure symmetric encryption scheme ( Enc $_{\text {sym }}$, Dec $\left._{\text {sym }}\right)$.

It outputs the global pubic parameter PP:

$$
\mathrm{PP}=\left\{\mathbb{G}, \mathbb{G}_{\mathrm{T}}, \mathrm{p}, \mathrm{e}, \mathrm{g}, \mathrm{H}, \mathrm{H}_{1}, \mathrm{H}_{2},\left(\mathrm{Enc}_{\text {sym }}, \operatorname{Dec}_{\text {sym }}\right)\right\}
$$

(2) Authorityset-up: each attribute authority performs this step to get their key pair. We take the A $A_{j}$ as an example

It chooses two random numbers $\alpha_{i}, \beta_{i} \in \mathbb{Z}_{p}^{*}$ for each attribute $\mathrm{i} \in S_{A, A A_{j}}$.

It chooses three random numbers $h_{j}, a_{j}, b_{j} \in \mathbb{Z}_{p}^{*}$.
It generates its pair of private key $\mathrm{sk}_{A A j}$ and public key $\mathrm{pk}_{\mathrm{AAj}}$ as follows:

$$
\left\{\begin{array}{l}
s k_{\mathrm{AAj}}=\left(\left\{\alpha_{i}, \beta_{i}\right\}_{i \in S_{\mathrm{AAj}}}, h_{j}, a_{j}, b_{j}\right), \\
p k_{\mathrm{AAj}}=\left(\left\{g^{\alpha_{i}}, g^{\beta_{i}}\right\}_{i \in S_{\mathrm{AAj}}}, g^{h_{j}}, g^{a_{j}}, g^{b_{j}}\right) .
\end{array}\right.
$$

5.1.2. Phase II: Encryption. We assume that the DO encrypts a message MSG with an self-defined access structure $\Psi$, and $S_{\Psi}$ is the attribute set which contains all attributes in the access structure $\Psi$. This phase contains three steps defined below:

\section{(1) Fully Hide the access policy}

It chooses a random number $\mathrm{a} \in \mathbb{Z}_{p}^{*}$ and then computes $\mathrm{q}_{i}=e\left(\left(g^{h_{j}}\right)^{a}, H(i)\right)$ where $\mathrm{i} \in S_{\Psi}$.

It replaces each attribute in $S_{\Psi}$ with the corresponding $\mathrm{q}_{i}$.

It converts the access policy to a LSSS access matrix ( $\left.\mathbb{M}_{l \times n}, \rho\right)$.

(2) Encrypt the key seed 
It chooses a random element $\mathrm{R} \in \mathbb{G}_{\mathrm{T}}$ (the key seed) to calculate $s=H_{1}(R, M S G)$ and the symmetric key Ksym $=H_{2}(R)$

It selects a $\mathrm{p}_{i} \in \mathbb{Z}_{p}$ for each row $\mathbb{M}_{i}$ of $\mathbb{M}$ and two random vectors $\vec{v}=\left[s, v_{1}, \cdots, v_{n}\right] \in \mathbb{Z}_{p}^{n}, \vec{w}=\left[0, w_{1}, \cdots, w_{n}\right] \in \mathbb{Z}_{p}^{n}$.

It computers $\lambda_{i}=\mathbb{M}_{i} \times \vec{v}$ and $\mathrm{w}_{i}=\mathbb{M}_{i} \times \vec{w}$.

It outputs the tuple $C T_{A B E}=\left(h,\left(\mathbb{M}_{l \times n}, \rho\right), C_{0}\right.$, $\left.\left\{C_{1, i}, C_{2, i}, C_{3, i}, C_{4, i}, C_{5, i}\right\}_{i \in[1, l]}\right)$ where $i$ presents a matrix row corresponding to an attribute.

Details of the ciphertext are presented as follows:

$$
\mathrm{CT}=\left\{\begin{array}{l}
h=g^{a}, \\
C_{0}=\operatorname{Re}(g, g)^{s}, \\
C_{1, i}=g^{\lambda_{i}} g^{\alpha_{\rho(i)} p_{i}}, \\
C_{2, i}=g^{p_{i}} \\
C_{3, i}=g^{w_{i}} g^{\beta_{\rho(i)} p_{i}}, \\
C_{4, i}=g^{a_{j} p_{i}} \\
C_{5, i}=g^{b_{j} p_{i}}
\end{array}\right.
$$

(3) Encrypt the message

Uses $\mathrm{K}_{\text {sym }}$ to encrypt the message MSG by the symmetric encryption algorithm $\mathrm{Enc}_{\text {sym }}$ and denote the result as $\mathrm{CT}_{\text {sym }}$ $=\mathrm{Enc}_{\mathrm{sym}}\left(\mathrm{K}_{\mathrm{sym}}, \mathrm{MSG}\right)$.

It uploads $\mathrm{CT}=\left\{\mathrm{CT}_{\mathrm{ABE}}, \mathrm{CT}_{\text {sym }}\right\}$ to the CS.

\subsubsection{Phase III: Key Generation}

\section{(1) Decryption key}

Each user owns an unique global identity GID $\in \mathbb{Z}_{p}^{*}$ and an attribute set $S_{\mathrm{GID}}$ where each attribute is associated with a designed attribute authority. Let $\mathrm{S}_{\mathrm{AA}, \mathrm{GID}}$ be the set of related attribute authorities. According to $\mathrm{S}_{\mathrm{AA}, \mathrm{GID}}$, we divide $\mathrm{S}_{\mathrm{GID}}$ into $\left\{S_{\mathrm{GID}, j}\right\}_{j \in S_{A A, G I D}}$. When the user queries its decryption key, each related AA runs the key generation algorithm. We take the $\mathrm{AA}_{j}$ as an instance.

It chooses a random number $r \in \mathbb{Z}_{p}^{*} \backslash\left\{-a_{j}+\mathrm{GID} / b_{j}\right\}$ for each $i \in S_{\mathrm{GID}, j}$.

It computes and returns the decryption key $s k_{\mathrm{GID}, j}=$ $\left\{K_{1, i}, K_{2, i}, K_{3, i}\right\}_{i \in S_{\mathrm{GID}}}$ :

$$
\left\{\begin{array}{l}
K_{1, i}=g^{\alpha_{i} / a_{j}+G I D+b_{j} r} H(G I D)^{\beta_{i} / a_{j}+G I D+b_{j} r}, \\
K_{2, i}=H(i)^{h_{j}} \\
K_{3, i}=r .
\end{array}\right.
$$

The decryption key of the user GID is noted as

$$
\mathrm{sk}_{\mathrm{GID}}=\left(\left\{\mathrm{sk}_{\mathrm{GID}, \mathrm{j}}\right\}_{\mathrm{j} \in \mathrm{S}_{\mathrm{AA}, \mathrm{GID}}}, \mathrm{GID}\right)=\left(\left\{\mathrm{K}_{1, \mathrm{i}}, \mathrm{K}_{2, \mathrm{i}}, \mathrm{K}_{3, \mathrm{i}}\right\}_{\mathrm{i} \in \mathrm{S}_{\mathrm{GID}}}, \mathrm{GID}\right)
$$

(2) Outsourced decryption key: the data user runs this algorithm

(a) Reconstructs the access policy

It computes $\mathrm{q}_{i}^{\prime}=e\left(h, H(i)^{h_{j}}\right)=e\left(g^{a}, H(i)^{h_{j}}\right), \forall i \in S_{\mathrm{GID}}$.

It uses $\mathrm{q}_{i}^{\prime}$ to replace the attribute $i$ to get the attribute set $\mathrm{S}_{\mathrm{GID}}^{\prime}$

It gains the access structure $\left(\mathbb{M}_{l \times n}, \rho\right)$ from $C T$.

It identifies the set of attributes $\mathrm{L}^{\prime}=\left\{i:\left(\rho(i) \cap S_{\mathrm{GID}}^{\prime}\right)_{i \in[l]}\right\}$ required for the decryption.

(b) Generates the outdec key

Chooses a random number $\mathrm{z} \in \mathbb{Z}_{p}^{*}$ to compute the outsourced decryption key $\left\{\mathrm{ok}_{\mathrm{GID}}\right\}=\left(\left\{\mathrm{opk}_{\mathrm{GID}}\right\}, \mathrm{osk}_{\mathrm{GID}}\right)$ as

$$
\left\{\begin{array}{l}
\operatorname{opk}_{\mathrm{GID}}=\left(\mathrm{GID},\left\{K_{1, \mathrm{i}}^{1 / z}, K_{3, i}\right\}_{i \in L^{\prime}}, g^{1 / \mathrm{z}}, \mathrm{H}(\mathrm{GID})^{1 / z}\right), \\
\mathrm{osk}_{\mathrm{GID}}=\mathrm{z}
\end{array}\right.
$$

\subsubsection{Phase IV: Decryption}

(1) Outsourced decryption : the CS performs outsourced decryption for the user

It computes the following equation for each matrix row corresponding to an attribute $i$ :

$Q=\frac{e\left(g^{1 / z}, C_{1, i}\right) e\left(H(\mathrm{GID})^{1 / z}, C_{3, i}\right)}{e\left(K_{1, i}^{1 / z} C_{2, i}^{\mathrm{GID}} C_{4, i} C_{5, i}^{K_{3, i}}\right)}=\left(e(g, g)^{\lambda_{i}} e(H(\mathrm{GID}), g)^{w_{i}}\right)^{1 / z}$.

It chooses a set of constants $\left\{c_{i}\right\}_{i \in[1, l]} \in \mathbb{Z}_{p}$ such that $\sum_{\mathrm{i}}$ $\mathrm{c}_{\mathrm{i}} \mathrm{M}_{\mathrm{i}}=[1,0, \cdots, 0]$.

It Computes

$$
\prod_{i=1}^{l} Q^{c_{i}}=\left(e(g, g) \sum_{i=1}^{l} \lambda_{i} c_{i} e(g, H(\mathrm{GID}))^{\sum_{i=1}^{l} w_{i} c_{i}}\right)^{1 / z},
$$

where $l$ is the row number of the access matrix. It returns $C T^{\prime}=\prod_{i=1}^{l} Q^{c_{i}}=e(g, g)^{s / z}$ to the user. 
(2) Uerdecryption: this phase contains the following two steps

(a) Recovers the message $\mathrm{R}$ based on the partially decrypted ciphertext $\mathrm{C} T^{\prime}$ by computing the following equation

$$
\mathrm{R}=\frac{C_{0}}{\left(C T^{\prime}\right)^{o s k}}=\frac{C_{0}}{\left(e(g, g)^{s / z}\right)^{z}}=\frac{C_{0}}{e(g, g)^{s}} .
$$

(note that this equation costs one exponentiation only and no pairing performance.)

(b) Computes $\mathrm{K}_{\text {sym }}=\mathrm{H}_{2}(\mathrm{R}), \quad \mathrm{MSG}=\operatorname{Dec}_{\text {sym }}\left(\mathrm{K}_{\text {sym }}, \mathrm{C}\right.$ $\left.\mathrm{T}_{\text {sym }}\right)$, and $\mathrm{s}=\mathrm{H}_{1}(\mathrm{R}, \mathrm{MSG})$

Judge if $\mathrm{CT}^{\prime}=\mathrm{e}(\mathrm{g}, \mathrm{g})^{s / z}$. If no, outputs $\perp$. Else, the user gains the right MSG.

Correctness of Equation (7):

Proof. First for each attribute $\mathrm{i} \in L^{\prime}$, the CS uses $\left(\left\{o p k_{G I D}\right\}\right.$ to compute:

$$
\begin{aligned}
\mathrm{Q} & =\frac{e\left(g^{1 / z}, g^{\lambda_{i}} g^{\alpha_{\rho(i)} p_{i}}\right) e\left(H(\mathrm{GID})^{1 / z}, g^{\beta_{\rho(i)} p_{i}} g^{w_{i}}\right)}{\left.\left.e\left(g^{\alpha_{\rho(i)} / z\left(a_{j}+G I D+b_{j} r\right.}\right) H(\mathrm{GID})^{\beta_{\rho(i)} / z\left(a_{j}+G I D+b_{j} r\right.}\right),\left(g^{p_{i}}\right)^{G I D} g^{a_{j} p_{i}} g^{b_{j} p_{i} r}\right)} \\
& =\frac{e(g, g)^{\lambda_{i} / z} e(g, g)^{\alpha_{\rho(i)} p_{i} / z} e(g, H(\mathrm{GID}))^{\beta_{\rho(i)} p_{i} / z} e(g, H(\mathrm{GID}))^{w_{i} / z}}{e(g, g)^{\alpha_{\rho(i)} p_{i} / z} e(g, H(\mathrm{GID}))^{\beta_{\rho(i)}} p^{p / z}} \\
& =e(g, g)^{\lambda_{i} / z} e(g, H(\mathrm{GID}))^{w_{i} / z} .
\end{aligned}
$$

Then, it chooses a set of constants $\left\{c_{i}\right\}_{i \in[1, l]} \in \mathbb{Z}_{p}$ such that $\sum_{\mathrm{i}} c_{i} \mathbb{M}_{i}=[1,0, \cdots, 0]$. Because $\lambda_{i}=\mathbb{M}_{i} \vec{v}$ and $\mathrm{w}_{i}=\mathbb{M}_{i} \vec{w}$, so

$$
\begin{aligned}
& \sum_{i=1}^{1} \lambda_{i} c_{i}=\sum_{i=1}^{1} \mathbb{M}_{i} \vec{v} c_{i}=\vec{v}[1,0, \cdots, 0]=s, \\
& \sum_{i=1}^{1} w_{i} c_{i}=\sum_{i=1}^{1} \mathbb{M}_{i} \vec{w} c_{i}=\vec{w}[1,0, \cdots, 0]=0 .
\end{aligned}
$$

Hence, we can get

$$
\begin{aligned}
\mathrm{C} T^{\prime} & =\prod_{i=1}^{l} Q^{c_{i}}=\prod_{i=1}^{l} e(g, g)^{c_{i} \lambda_{\rho(i)} / z} e(g, H(\mathrm{GID}))^{c_{i} w_{i} / z} \\
& =e(g, g) \sum_{i=1}^{l} c_{i} \lambda_{\rho(i)} l z e(g, H(\mathrm{GID}))_{i=1}^{l} c_{i} w_{i} / z \\
& =e(g, g)^{s / z} e(g, H(\mathrm{GID}))^{0}=e(g, g)^{s / z}
\end{aligned}
$$

Then, based on $C T^{\prime}$, the user recovers

$$
R=\frac{C_{0}}{\left(C T^{\prime}\right)^{o s k}}=\frac{\operatorname{Re}(g, g)^{s}}{\left(e(g, g)^{s / z}\right)^{z}}=\frac{\operatorname{Re}(g, g)^{s}}{e(g, g)^{s}} .
$$

5.1.5. Phase V: Trace. The Trace(PP, $\left.s k_{\mathrm{GID}},\left\{p k_{\mathrm{AAj}}\right\}\right)$ algorithm is performed by all attribute authorities. The input is the private key sk $\mathrm{GID}_{\mathrm{GI}}=\left(\left\{\mathrm{sk}_{\mathrm{GID}, \mathrm{j}}\right\}_{\mathrm{j} \in \mathrm{S}_{\mathrm{AA}}^{\prime}}, \mathrm{GID}\right)=\left(\left\{\mathrm{K}_{1, \mathrm{i}}, \mathrm{K}_{2, \mathrm{i}}, \mathrm{K}_{3, \mathrm{i}}\right\}_{\mathrm{i} \in \mathrm{S}_{\mathrm{GID}}}, \mathrm{GID}\right)$ of a user.

Firstly, the AA checks the form of the key. If the key does not satisfies the form, this algorithm aborts.

Then, the AA searches its database to find if $\exists i \in S_{G I D}$, s.t.

$$
\begin{gathered}
\mathrm{K}_{1, i}, K_{2, i} \in \mathbb{G}, K_{3, i}, G I D \in \mathbb{Z}_{p}^{*}, \\
\mathrm{e}\left(K_{1, i}, g^{a_{j}} g^{\left(b_{j}\right)^{K_{3, i}}} g^{\mathrm{GID}}\right)=e(g, g)^{\alpha_{i}} e\left(H(\mathrm{GID}), g^{\beta_{i}}\right) .
\end{gathered}
$$

If yes, the global identity GID of the guilty user will be output.

5.2. Application in the EHR System. In this section, we describe a simple application of our scheme based on the electronic health record (EHR) system. The basic procedures are presented in Figure 6, and the details are described as follows:

(1) The central trusted authority (the government) performs the system set-up algorithm to generate and publish the global parameters PP

(2) A set of management companies act as attribute authorities, and each attribute authority needs to set up first. Then, they publish their public keys while keeping private keys secret

(3) A hospital encrypts a patient's medical records Rd based on a user-defined access structure $\mathbb{M}$ and sends the ciphertext $\mathrm{CT}$ along with the fully hidden access structure $n$ to the cloud storing server to store

$$
\text { Hospital } \stackrel{\text { Encrypt(Rd,M) } \rightarrow \text { CT }}{\longrightarrow} \text { Cloud. }
$$

(4) Before a data user (a doctor) requests the wanted records from the cloud server, he/she needs to get the decryption key $s k_{\mathrm{GID}}$ from the attribute authorities first

(5) To outsource the decryption work to the cloud server, the doctor generates the outsourced decryption key o $p k_{\mathrm{GID}}$ based on $s k_{\mathrm{GID}}$. Then, he/she sends op $k_{\mathrm{GID}}$ to the cloud server

(6) The cloud server will partially decrypt for the doctor as long as his/her attribute set satisfies the encryption 


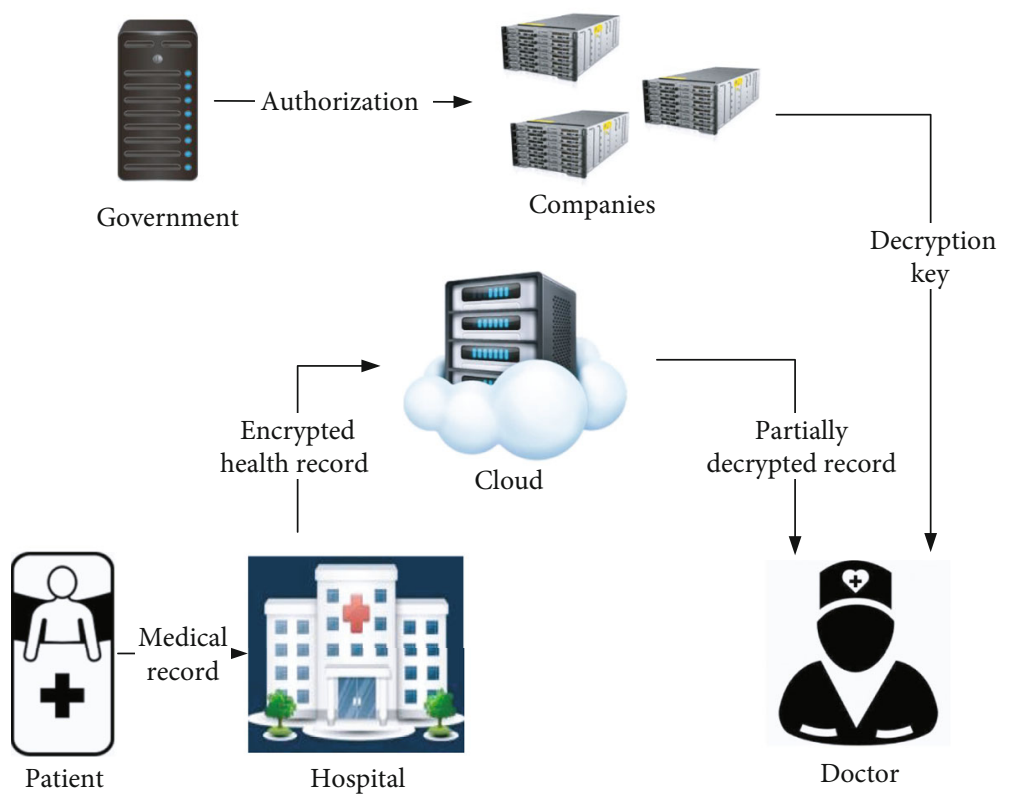

Figure 6: Application in an EHR system.

access structure. Then, the cloud sends the partially decrypted ciphertext $C T^{\prime}$ back to the doctor

Cloud $\longrightarrow$ Decrypt $_{\text {out }}\left(\mathrm{opk}_{\mathrm{GID}}, \mathrm{CT}\right) \rightarrow \mathrm{CT} \longrightarrow{ }^{\prime}$ Doctor.

(7) Finally, the doctor can fully decrypt and get the medical records. Note that doctors only require one exponentiation in $\mathbb{G}_{T}$ to fully decrypt, and one hash operation to verify whether the ciphertext was tampered

$$
\operatorname{Decrypt}\left(\mathrm{CT}^{\prime}\right) \rightarrow \mathrm{Rd} \text {. }
$$

(8) If a malicious user decrypt illegally with a valid decryption key, the attribute authorities can perform the trace algorithm to recover the identity of the guilty user who leaks his/her decryption key to a illegal user

\section{Security Analysis}

\subsection{Indistinguishability}

Theorem 1. If Lewko et al.'s scheme [8] is CPA=secure, our multiauthority attribute-based encryption scheme is selectively replayable CCA-secure according to Definition 6 such that $A d v_{A}\left[\operatorname{Exp}^{\operatorname{Conf}}\right]<A d v_{A}\left[\operatorname{Exp}^{\text {Lewko }}\right]$.
Proof. We define a PPT adversary $\mathbb{A}$ running the experiment defined in Section 4.3(1) with an entity $\mathbb{B}$. $\mathbb{B}$ running Lewko et al.'s CPA-secure [8] experiment with a challenger $\mathbb{C}$. The proof described below is going to show that the advantage of $A$ to win the experiment Exp ${ }^{\text {Conf }}$ is smaller than the advantage of $\mathbb{B}$ to win Lewko et al.'s CPA-secure experiment Ex $\mathrm{p}^{\text {Lewko }}$. The detailed interactions are described as follows:

(1) Initialization: the adversary $\mathbb{A}$ submits a challenge access policy $\Psi^{*}=\left(\mathbb{M}^{*}, \rho^{*}\right)$ to the challenge $\mathbb{C}$ through $\mathbb{B}$

(2) Set-Up: $\mathbb{C}$ runs the $\operatorname{Setup}(\lambda)$ algorithm to generate the global parameter PP

It chooses two multiplicative cyclic groups $\mathbb{G}, \mathbb{G}_{\mathrm{T}}$ of prime order $\mathrm{p}$ with a generator $\mathrm{g}$ of $\mathbb{G}$.

It chooses a bilinear map e $: \mathbb{G} \times \mathbb{G} \rightarrow \mathbb{G}_{\mathrm{T}}$.

It chooses three collusion-resistant hash functions $\mathrm{H}^{*}$ $:\{0,1\}^{*} \rightarrow \mathbb{G}, \mathrm{H}_{1}^{*}:\{0,1\}^{*} \rightarrow \mathbb{Z}_{\mathrm{p}}^{*}, \mathrm{H}_{2}^{*}:\{0,1\}^{*} \rightarrow\{0,1\}^{\mathrm{k}}$.

It chooses a cpa-secure symmetric encryption scheme ( Enc $_{\text {sym }}$, Dec $_{\text {sym }}$ ).

It sends the global parameter $\mathrm{PP}=\left\{\mathbb{G}, \mathbb{G}_{\mathrm{T}}, \mathrm{p}, \mathrm{e}, \mathrm{g}, \mathrm{H}^{*}\right.$, $\left.\mathrm{H}_{1}^{*}, \mathrm{H}_{2}^{*},\left(\mathrm{Enc}_{\text {sym }}, \operatorname{Dec}_{\text {sym }}\right)\right\}$ to $\mathbb{A}$ through $\mathbb{B}$.

It runs the Setup ${ }_{\text {auth }}$ algorithm to generate the key pairs of the noncorrupted authorities:

It chooses two random numbers $\alpha_{\mathrm{i}}$ and $\beta_{\mathrm{i}} \in \mathbb{Z}_{\mathrm{p}}^{*}$ for each attribute $\mathrm{i} \in \mathrm{S}_{\mathrm{A}, \mathrm{AA}_{\mathrm{j}}}$.

It chooses three random numbers $h_{j}, a_{j}$, and $b_{j} \in \mathbb{Z}_{p}^{*}$ to compute the public key $\mathrm{pk}_{\mathrm{AA}_{\mathrm{j}}}=\left(\left\{\mathrm{g}^{\alpha_{\mathrm{i}}}, \mathrm{g}^{\beta_{\mathrm{i}}}\right\}_{\mathrm{i} \in \mathrm{S}_{\mathrm{A}, \mathrm{AA}}}, \mathrm{g}^{\mathrm{h}_{\mathrm{j}}}, \mathrm{g}^{\mathrm{a}_{\mathrm{j}}}, \mathrm{g}^{\mathrm{b}_{j}}\right)$.

It sends all attribute authorities' public keys to $\mathbb{A}$ through $\mathbb{B}$.

A runs the Setup ${ }_{\text {auth }}$ algorithm to generate the key pairs of the corrupted authorities in the same way. 
(3) Query phase I: $\mathbb{B}$ initializes three empty tables $\mathbb{T}, \mathbb{T}_{1}$, $\mathbb{T}_{2}$, an empty set $\mathbb{D}$, and an integer $\mathrm{j}=0$. Details of queries are described as follows:

\section{(a) Hash query}

$\mathrm{H}_{1}^{*}$ oracle: if the entry ( $\left.\mathrm{R}, \mathrm{MSG}, \mathrm{s}\right)$ already existed in Table $\mathbb{T}_{1}$, return $s$. Otherwise, it chooses a random element $s \in \mathbb{Z}_{\mathrm{p}}^{*}\left(s\right.$ is unique in Table $\left.\mathbb{T}_{1}\right)$. Then, it records (R, MSG, $s$ ) in Table $\mathbb{T}_{1}$ and returns s.

$\mathrm{H}_{2}^{*}$ oracle: if the entry $\left(\mathrm{R}, \mathrm{K}_{\text {sym }}\right)$ already existed in Table $\mathbb{T}_{2}$, return $K_{\text {sym }}$. Otherwise, it chooses a random element $\mathrm{K}_{\text {sym }} \in\{0,1\}^{\mathrm{k}}$. Then, it records $\left(\mathrm{R}, \mathrm{K}_{\text {sym }}\right)$ in Table $\mathbb{T}_{2}$ and returns $\mathrm{K}_{\text {sym }}$.

(b) Key query: In the q-th query, $\mathbb{A}$ queries the decryption key related with an attribute set $\mathrm{S}_{\mathrm{GID}_{\mathrm{q}}}$ by sending $\mathrm{S}_{\mathrm{GID}_{\mathrm{q}}}$ and $\mathrm{GID}_{\mathrm{q}}$ to $\mathbb{B}$. $\mathbb{B}$ calls $\mathbb{C}$ to generate the decryption key and sends it to $\mathbb{A}$. $\mathbb{C}$ chooses a random number $r \in \mathbb{Z}_{\mathrm{p}}^{*} \backslash\left\{-\mathrm{a}_{\mathrm{j}}+\mathrm{GID}_{\mathrm{q}} / \mathrm{b}_{\mathrm{j}}\right\}$ to compute the decryption key $\mathrm{sk}_{\mathrm{GID}_{\mathrm{q}}}=\left(\left\{\mathrm{sk}_{\mathrm{GID}_{\mathrm{q}}, \mathrm{j}}\right\}_{\mathrm{j} \in \mathrm{S}_{\mathrm{AA}, \mathrm{GID}}}, \mathrm{GID}\right)$ $=\left(\left\{\mathrm{K}_{1, \mathrm{i}}, \mathrm{K}_{2, \mathrm{i}}, \mathrm{K}_{3, \mathrm{i}}\right\}_{\mathrm{i} \in \mathrm{S}_{\mathrm{GID}}}, \mathrm{GID}\right)$ while setting $\mathbb{D}=\mathbb{D} \cup$ $\mathrm{S}_{\mathrm{GID}_{\mathrm{q}}}$

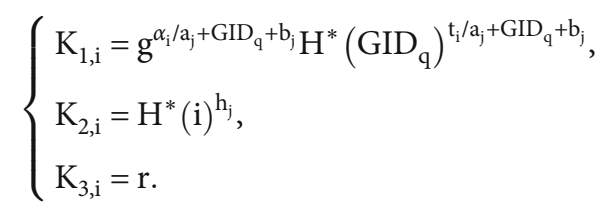

$\mathbb{B}$ chooses a random element $a \in \mathbb{Z}_{p}^{*}$ to compute $h=g^{a}$ to simulate the output of the encryption algorithm. $\mathbb{B}$ calls $\mathbb{C}$ to run the outsourced decryption key generation algorithm: $\mathbb{C}$ chooses a random number $\mathrm{z} \in \mathbb{Z}_{\mathrm{p}}^{*}$ to compute

$$
\left\{\begin{array}{l}
\operatorname{opk}_{\mathrm{GID}_{\mathrm{q}}}=\left(\left\{\mathrm{K}_{1, \mathrm{i}}^{1 / z}\right\}_{\mathrm{i} \in \mathrm{L}^{\prime}}, \mathrm{g}^{1 / z}, \mathrm{H}^{*}(\mathrm{GID})^{1 / z}\right), \\
\operatorname{osk}_{\mathrm{GID}_{\mathrm{q}}}=\mathrm{z} .
\end{array}\right.
$$

Sends $\mathrm{ok}_{\mathrm{GID}_{\mathrm{q}}}=\left(\mathrm{opk}_{\mathrm{GID}_{\mathrm{q}}}, \mathrm{osk}_{\mathrm{GID}_{\mathrm{q}}}\right)$ to $\mathbb{B}$. $\mathbb{B}$ stores the entry $\left(\mathrm{q}, \mathrm{S}_{\mathrm{GID}_{\mathrm{q}}}, \mathrm{sk}_{\mathrm{GID}_{\mathrm{q}}}, \mathrm{ok}_{\mathrm{GID}_{\mathrm{q}}}\right)$ in the table $\mathbb{T}$. Finally, $\mathbb{B}$ returns the key to $\mathbb{A}$.

(c) Decryption query: without loss of generality, we assume that all ciphertexts input to this query have been partially decrypted. For instance, we assume that $\mathrm{CT}^{\prime}$ was correctly decrypted by opk of the entry $\left(\mathrm{q}, \mathrm{S}_{\mathrm{GID}_{\mathrm{q}}}, \mathrm{sk}_{\mathrm{GID}_{\mathrm{q}}}, \mathrm{ok}_{\mathrm{GID}_{\mathrm{q}}}\right)$. Let $\mathrm{CT}^{\prime}$ be associated with a structure $(\mathbb{M}, \rho)$ which is not equal with $\left(\mathbb{M}^{*}, \rho^{*}\right)$. Let opk be associated with a set of attributes which satisfies $(\mathbb{M}, \rho)$ and not satisfies $\left(\mathbb{M}^{*}, \rho^{*}\right)$
TABLe 3: Notations.

\begin{tabular}{lr}
\hline Notation & Meaning \\
\hline $\mathrm{E}, / E_{T}$ & One exponentiation in group $\mathbb{G} / \mathbb{G}_{T}$ \\
$P_{e}$ & One pairing operation of the pairing function $e$. \\
$N_{e}$ & The row number of the encryption LSSS access matrix. \\
$N_{\mathrm{u}}$ & The attribute number of the user attribute set. \\
$N_{\mathrm{d}}$ & The attribute number required in decryption. \\
$N_{a}$ & The attribute number of the attribute universe set. \\
\hline
\end{tabular}

Search Table $\mathbb{T}_{1}$ to find if there exists an entry ( $R$, MSG , s) which satisfies $\left(\mathrm{CT}^{\prime}\right)^{\text {osk }_{\mathrm{GI}_{\mathrm{q}}}}=\mathrm{e}(\mathrm{g}, \mathrm{g})^{\mathrm{s}}$. If not, abort. Else, obtain entry $\left(R, K_{\text {sym }}\right)$ in Table $\mathbb{T}_{2}$. If this entry does not exist, abort. Else, test if $\mathrm{C}_{0}=\operatorname{Re}(\mathrm{g}, \mathrm{g})^{\mathrm{s}}$ and $\mathrm{CT}_{\text {sym }}=\mathrm{Enc}_{\text {sym }}\left(\mathrm{K}_{\text {sym }}\right.$, MSG). If yes, output MSG. Else, abort.

(4) Challenge: $A$ chooses two message $\mathrm{MSG}_{1}, \mathrm{MSG}_{2} \in$ $\{0,1\}^{*}$ with same length then sends them to $\mathbb{B}$. $\mathbb{B}$ chooses two message $R_{0}, R_{1} \in \mathbb{G}_{T}$ with same length and then sends them to $\mathbb{C}$. $\mathbb{C}$ chooses a random bit $\mathrm{b} \in\{0,1\}$, then $\mathbb{C}$ encrypts $R_{b}$ under the access structure $\left(\mathbb{M}^{*}, \rho^{*}\right)$ by running Lewko's scheme. Finally, $\mathbb{C}$ returns $\mathrm{CT}_{\mathrm{b}, \mathrm{ABE}}^{*}$ to $\mathbb{B}$. $\mathbb{B}$ guesses $b$ with advantage $\operatorname{Adv}_{\mathrm{A}}\left[\operatorname{Exp}^{\text {Lewko }}\right]$. Then, $\mathbb{B}$ computes $\mathrm{K}_{\text {sym }}^{*}=\mathrm{H}_{2}^{*}\left(\mathrm{R}_{\mathrm{b}}^{*}\right)$ and $\mathrm{CT}_{\mathrm{b}, \mathrm{sym}}^{*}=\mathrm{Enc}_{\mathrm{sym}}\left(\mathrm{K}_{\mathrm{sym}}^{*}, \mathrm{MSG}_{\mathrm{b}}\right)$. Finally, $\mathbb{B}$ returns $\mathrm{CT}_{\mathrm{b}}^{*}=\left(\mathrm{CT}_{\mathrm{b}, \mathrm{ABE}}^{*}, \mathrm{CT}_{\mathrm{b}, \mathrm{sym}}^{*}\right)$ to $\mathrm{A}$

(5) Query phase II: the adversary $\mathbb{A}$ can query a polynomially bounded number of queries as in query phase II after receiving the ciphertext $C T_{b}^{*}$ with restrictions that the queried attribute set cannot satisfy the challenge access structure, and the response of the decryption query cannot be either $\mathrm{MSG}_{0}$ or $\mathrm{MSG}_{1}$

(6) Guess: $\mathbb{A}$ tries to guess $b^{\prime}$ based on $C T_{b}^{*}$. Then, $\mathbb{A}$ sends $b^{\prime}$ to $\mathbb{C}$ through $\mathbb{B}$. If $b^{\prime}=b$, we say that $\mathbb{A}$ wins this experiment

We can easily get that the advantage of $\mathbb{A}$ to win the experiment $\operatorname{Exp}^{\text {conf }}$ is smaller than the advantage of $\mathbb{B}$ to win the experiment Exp ${ }^{\text {Lewko }}$, because $\mathbb{A}$ has to be based on the right $C T_{b}^{*}$ provided by $\mathbb{B}$ to guess $b$ successfully. In other words, $\operatorname{Pr}\left[\operatorname{Exp}_{\mathrm{A}^{\text {Lewko }}}\left(1^{\xi}\right)\right]>\operatorname{Pr}\left[\operatorname{Exp}_{\mathrm{A}}\right.$ Conf-Real $\left.\left(1^{\xi}\right)\right]$ and our scheme achieve selectively replayable CCA secure.

\subsection{Verifiability}

Theorem 2. If $H_{1}$ and $H_{2}$ are two collision-resistant hash functions, our scheme is verifiable against malicious servers.

Proof. We define a PPT adversary $\mathbb{A}$ running the experiment defined in Section 4.3(2) with an entity $\mathbb{B}$. $\mathbb{B}$ tries to break the collision resistance of the two hash functions $\mathrm{H}_{1}^{*}$ and $\mathrm{H}_{2}^{*}$.

(1) Initialization: the adversary $\mathbb{A}$ submits a challenge access policy $\Psi^{*}=\left(\mathbb{M}^{*}, \rho^{*}\right)$ to the entity $\mathbb{B}$ 
TABLE 4: Storage cost comparison.

\begin{tabular}{lcccc}
\hline Scheme & {$[11]$} & {$[13]$} & {$[14]$} & Our scheme \\
\hline Decryption key length & $2 N_{u}|\mathbb{G}|$ & $3 N_{u}|\mathbb{G}|+N_{u}\left|\mathbb{Z}_{p}^{*}\right|+\left|\mathbb{Z}_{p}\right|$ & $\left(2 N_{u}+3\right)|\mathbb{G}|+2\left|\mathbb{Z}_{p}\right|$ & $2 N_{u}|\mathbb{G}|+N_{u}\left|\mathbb{Z}_{p}\right|$ \\
Outdec key length & $\left(N_{d}+2\right)|\mathbb{G}|+\left|\mathbb{Z}_{p}^{*}\right|$ & - & $\left(2 N_{u}+2\right)|\mathbb{G}|$ & $\left(N_{d}+2\right)|\mathbb{G}|+\left|\mathbb{Z}_{p}^{*}\right|$ \\
Ciphertext length & $\left(3 N_{e}+1\right)|\mathbb{G}|+1\left|\mathbb{G}_{T}\right|$ & $5 N_{e}|\mathbb{G}|+\left(N_{e}+1\right)\left|\mathbb{G}_{T}\right|$ & $\left(3 N_{e}+2\right)|\mathbb{G}|+1\left|\mathbb{G}_{T}\right|$ & $\left(5 N_{e}+1\right)|\mathbb{G}|+1\left|\mathbb{G}_{T}\right|$ \\
\hline
\end{tabular}

(2) Set-up: $\mathbb{B}$ runs the Setup algorithm to generate the global parameter except the two hash functions

(3) $\mathbb{B}$ runs the Setup auth algorithm to generate keypairs of attribute authorities.

Query: A runs the adversary queries as defined in query phase I and query phase II through $\mathbb{B}$ to get the related decryption keys and outsourced decryption keys

(4) Challenge: $\mathbb{A}$ sends the challenge message $\mathrm{MSG}^{*}$ to $\mathbb{B}$ , and $\mathbb{B}$ answers as follows

It chooses a random message $R^{*} \in G_{T}$ to run Lewko's encryption scheme to encrypt $R^{*}$ under the access policy ( $\left.\mathbb{M}^{*}, \rho^{*}\right)$.

It computes $s^{*}=H_{1}^{*}\left(R^{*} \| \mathrm{MSG}^{*}\right)$ and $K_{\text {sym }}^{*}=H_{2}^{*}\left(R^{*}\right)$.

It runs the symmetric encrypt algorithm to encrypt MS $\mathrm{G}^{*}$ to generate the ciphertext $\mathrm{CT}^{*}=\left(\mathrm{CT}_{\mathrm{ABE}}^{*}, \mathrm{CT}_{\text {sym }}^{*}\right)=(\mathrm{h}$, $\left.\mathrm{C}_{0},\left\{\mathrm{C}_{1, \mathrm{i}}, \mathrm{C}_{2, \mathrm{i}}, \mathrm{C}_{3, \mathrm{i}}, \mathrm{C}_{4, \mathrm{i}}, \mathrm{C}_{5, \mathrm{i}}\right\}, \mathrm{CT}_{\text {sym }}^{*}\right)$.

It returns the ciphertext $\mathrm{CT}^{*}=\left(\mathrm{CT}_{\mathrm{ABE}}^{*}, \mathrm{CT}_{\text {sym }}^{*}\right)$ to $\mathbb{A}$.

If $\mathbb{B}$ can recover a message $M S G \in\left\{\mathrm{MSG}^{*}, \perp\right\}$, then we say A wins this experiment. Hence there are two cases are considered:

(1) $(\mathrm{MSG}, \mathrm{R}) \neq\left(\mathrm{MSG}^{*}, \mathrm{R}^{*}\right)$, which means that $\mathbb{B}$ finds a collision of the hash function $H_{1}^{*}$

(2) $\left(\mathrm{K}_{\text {sym }}, \mathrm{CT}_{\text {sym }}\right)=\left(\mathrm{K}_{\text {sym }}^{*}, \mathrm{CT}_{\text {sym }}^{*}\right)$ but $\left(\mathrm{R}^{*} \neq \mathrm{R}\right)$, which means that $\mathbb{B}$ breaks the collision resistance condition of $H_{2}^{*}$ such as $H_{2}^{*}(\mathrm{R})=K_{\text {sym }}=K_{\text {sym }}^{*}=H_{2}^{*}\left(R^{*}\right)$

In other words, since $\mathrm{H}_{1}$ and $\mathrm{H}_{2}$ are two collisionresistant hash functions, the outsourced decryption of our scheme is verifiable.

\subsection{Fully Hiding}

Theorem 3. Our scheme is an outsourced ABE with fully hidden policy if the one-way anonymous key agreement protocol [15] is IND-CPA secure.

Proof. The purpose of this proof is that no PPT adversary can recover the access policy without the right decryption key. The setup phase and the query phase 1 are same as the confidentiality experiment.

In the challenge phase, the adversary $\mathbb{A}$ chooses two challenge messages $R_{0}^{*}, R_{1}^{*}$ and two valid access policies $\Psi_{0}, \Psi_{1}$, and then it sends them to the challenger $\mathbb{C}$. Notice, $\Psi_{0}$ and $\Psi_{1}$ satisfy the following restriction: either all the attribute sets

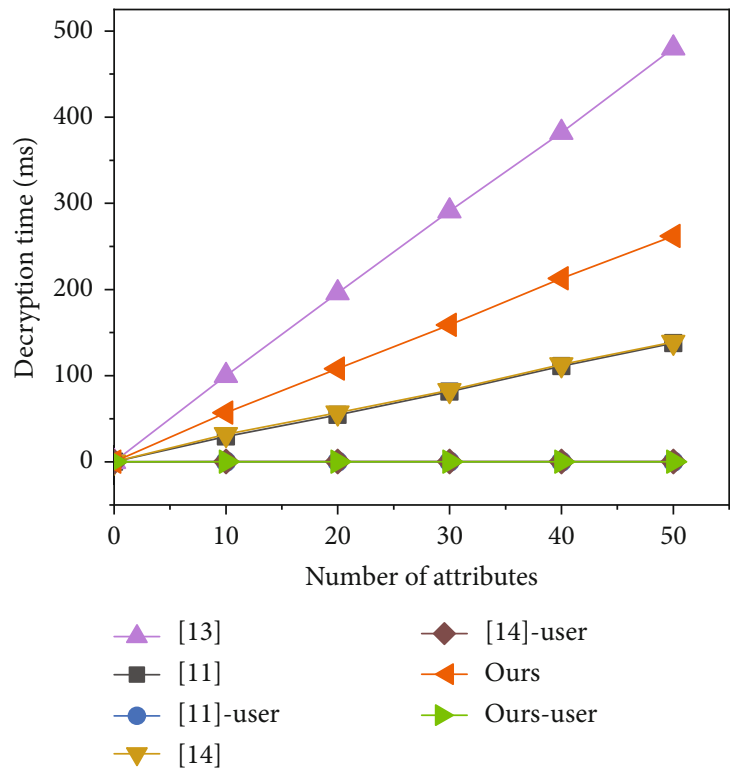

Figure 7: Decryption cost.

queried in query phase 1 satisfy none of the policy or all attribute sets satisfy both the policies. Then, $\mathbb{C}$ computes $q^{\prime}(i)$ $=e\left(\left(g^{h_{j}}\right)^{a}, H(i)\right)$ based on the one-way anonymous key agreement protocol where $a \in \mathbb{Z}_{p}^{*}$ is a random number. This step is used to hide the real policy by replacing each attributes in the policy with the corresponding $q^{\prime}(i)$. Then, $\mathbb{C}$ chooses a random bit $b \in\{0,1\}$ and encrypts the message $R_{b}^{*}$ under the access policy $\Psi_{b}$. Finally, $\mathbb{C}$ sends $C T^{*}$ to $\mathbb{A}$. After that, $\mathbb{A}$ still can query a polynomially bounded number of queries as in query phase I. The none-or-both principle still works in this phase.

In the guess phase, $\mathbb{A}$ outputs $b^{\prime}$.

When $A$ tries to decrypt $C T^{*}$, it has to recover the access policy first. In our scheme, the decryption key $\mathrm{K}_{2, i}=H(i)^{h_{j}}$ is necessary for it to compute $q^{\prime}(i)$ because we computed the $\mathrm{q}^{\prime}(i)$ based on the one-way anonymous key agreement protocol before we encrypted the message. It means only the authorized user can get the right access policy. And due to the random value $a$, unauthorized user cannot guess attribute $i$ from $q^{\prime}(i)$ which prevents the collusion of the users. Hence, the advantage of the adversary to win the experiment $\mathrm{A} d v_{A}$ [ $\left.\operatorname{Exp}^{\text {Priv }}\left(1^{\xi}\right)\right]$ is negligible, and our scheme ensures the privacy preservation of the access policy against adaptive chosen plaintext attack. 
6.4. Traceability. In this section, we prove that our scheme is fully traceable under the $q-\mathrm{SDH}$ assumption.

Lemma 1. Our scheme achieves fully user traceability based on that the Boneh-Boyen fully signature scheme [31] is strong existential forgery secure against adaptive chosen message attack.

Proof. We define a PPT adversary $\mathbb{A}$ running the experiment defined in Section 4.3(4) to attack our scheme through an entity $\mathbb{B}$ by $\mathbb{B}$ breaking the Boneh-Boyen fully signature scheme with the same advantage under adaptive chosen message attacks. Assuming the advantage of the adversary $\mathbb{A}$ to break our scheme is $\varepsilon$, and $\mathbb{B}$ can access a random oracle $H$. Let $\mathbb{C}$ be the challenger in the B-B scheme, Sig $\in \mathbb{G}$ be the signature of $\mathrm{A} A_{j}$, and $\mathrm{p} k_{A A_{j}}^{s i g}=\left\{\mathbb{G}, p, g, g^{a_{j}}, g^{b_{j}}\right\}$ is the associated public key of $\mathrm{Sig}_{j}$.

(1) Set-up: the challenger $\mathbb{C}$ runs the Setup algorithm to generate the global parameter PP and sends PP to $\mathbb{B}$ . For each noncorrupted authorities in the set $S^{\prime}, \mathbb{C}$ sends $\mathrm{p} k_{A A j}^{s i g}$ to $\mathbb{B}$. Then, $\mathbb{B}$ chooses two random numbers $\alpha_{i}, \beta_{i}$ for each attribute in the attribute set of the authority, and then $\mathbb{B}$ chooses a random number $h_{j}$ to generate the public key of the authority $\mathrm{p} k_{\mathrm{AA} j}=($ $\left.\left\{g^{\alpha_{i}}, g^{\beta_{i}}\right\}_{i \in S_{\mathrm{A}, \mathrm{AA}}}, g^{h_{j}}, g^{a_{j}}, g^{b_{j}}\right)$. Finally, $\mathbb{B}$ returns PP and $\left\{p k_{A \mathrm{~A} j}\right\}_{j \in S^{\prime}}$ to $\mathbb{A}$. For corrupted authorities, $\mathbb{A}$ runs the Setup $p_{\text {auth }}$ algorithm to generate the key pairs for them

(2) Key query: $\mathbb{A}$ runs $m$ queries. In the $q$-th query, $\mathbb{A}$ sends $\left(S_{\mathrm{GID}_{q}}, \mathrm{GID}_{q}\right)$ to $\mathbb{B}$. $\mathbb{B}$ initiates an empty table $\mathbb{}$ and do the following steps

(a) Accesses the random oracle $H\left(\mathrm{GID}_{q}\right)$ : $\mathbb{B}$ searches the entry $\left(\mathrm{GID}_{\mathrm{q}}, t_{\mathrm{GID}_{q}}, g^{t_{\mathrm{GID}_{q}}}\right)$ in the table $\mathbb{T}$, and if it exists, $\mathbb{B}$ outputs $\mathrm{g}^{t_{\mathrm{GID}_{q}}}$. Else, $\mathbb{B}$ chooses a random number $\mathrm{t}_{\mathrm{GID}_{q}}$ while stores $\left(\mathrm{GID}_{q}, t_{\mathrm{GID}_{q}}, g^{t_{\mathrm{GID}_{q}}}\right)$ in the table $\mathbb{T}$. $\mathbb{B}$ outputs $\mathrm{g}^{t_{\mathrm{GID}_{q}}}$

(b) Generates the decryption key $s k_{\mathrm{GID}_{q, j}}: \mathbb{C}$ chooses a random number $r \in \mathbb{Z}_{p}^{*} \backslash\left\{-a_{j}+\mathrm{GID}_{q} / b_{j}\right\}$ for each attribute $\mathrm{i} \in S_{\mathrm{GID}_{q}, j}$ and returns the signature $(r, \sigma=$ $\left.g^{1 / a_{j}+G I D_{q}+b_{j} r}\right)$. Then, $\mathbb{B}$ computes the components of $s k_{\mathrm{GID}_{q}, j}$

$$
\left\{\begin{array}{l}
K_{1, i}=\sigma^{\left(\alpha_{i}+\beta_{i} t_{G I D_{q}}\right)}=g^{\alpha_{i}+\beta_{i} t_{G I D_{q}} / a_{j}+G I D_{q}+b_{j} r}=g^{\alpha_{i} / a_{j}+G I D_{q}+b_{j} r} g^{t_{G I D_{q}} \beta_{i} / a_{j}+G I D_{q}+b_{j} r=g^{\alpha_{i} / a_{j}+G I D_{q}+b_{j} r} H\left(G I D_{q}\right)^{\beta_{i} / a_{j}+G I D_{q}+b_{j} r}} \\
K_{2, i}=H(i)^{h_{j}} \\
K_{3, i}=r .
\end{array}\right.
$$

Then, $\mathbb{B}$ sets $\mathbb{D}=\mathbb{D} \cup S_{\mathrm{GID}_{q}}$. Finally $\mathbb{B}$ returns the following result to $\mathbb{A}$ :

$s k_{G I D_{q}}=\left(\left\{s k_{G I D_{q}, j}\right\}_{j \in S_{A A, G I D}}, G I D_{q}\right)=\left(\left\{K_{1, i}, K_{2, i}, K_{3, i}\right\}_{i \in S_{G I D_{q}}}, G I D_{q}\right)$.

(3) Key forgery: $\mathbb{A}$ sends a $s k^{*}$ to $\mathbb{B}$. The advantage of the adversary to win is defined as

$$
\operatorname{Pr}\left[\operatorname{Trace}\left(P P,\left\{p k_{\mathrm{AAj}}\right\}, \mathrm{sk}^{*}\right) \in\left\{\perp, G I D_{1}, \cdots, G I D_{m}\right\}\right]=\varepsilon,
$$

where $\left(\mathrm{GID}_{1}, \cdots, \mathrm{GID}_{m}\right)$ is $m \mathrm{GID}$ queried in the last phase. If $\operatorname{Trace}\left(\mathrm{PP},\left\{p k_{\mathrm{AAj}}\right\}, s k^{*}\right) \in\left\{\perp, \mathrm{GID}_{1}, \cdots, \mathrm{GID}_{m}\right\}$, it means $s k^{*}$ $=\left(\left\{K_{1, i}, K_{2, i}, K_{3, i}\right\}_{i \in \mathrm{S}_{\mathrm{GID}}}\right.$, GID) passed the form check and
$\mathrm{GID} \in\left\{\perp, \mathrm{GID}_{1}, \cdots, \mathrm{GID}_{m}\right\}$. Hence, $\exists i \in S$, s.t.

$$
\begin{gathered}
\mathrm{K}_{1, i}, K_{2, i} \in \mathbb{G}, K_{3, i}, \mathrm{GID} \in \mathbb{Z}_{p}^{*}, \\
\mathrm{e}\left(K_{1, i}, g^{a_{j}} g^{\left(b_{j}\right)^{K_{3, i}}} g^{G I D}\right)=e(g, g)^{\alpha_{i}} e\left(H(\mathrm{GID}), g^{t_{i}}\right) .
\end{gathered}
$$

Without loss of generality, we assume the adversary $\mathbb{A}$ accessed the random oracle $H$ (GID) before it outputs the $s$ $k^{*}$. B obtains the entry (GID, $t_{\mathrm{GID}}, g^{t_{\mathrm{GID}}}$ ) from the table $\mathbb{T}$. According to $\mathrm{e}\left(K_{1, i}, g^{a_{j}} g^{\left(b_{j}\right)^{K_{3, i}}} g^{\mathrm{GID}}\right)=e(g, \mathrm{~g})^{\alpha_{i}} e(H(\mathrm{GID})$, $\left.g^{\beta_{i}}\right)$, we can get $\mathrm{K}_{1, i}=g^{\alpha_{i}+t_{\mathrm{GID}} \beta_{i} / a_{j}+b_{j} K_{3, i}+\mathrm{GID}}$. Then, $\mathbb{B}$ computes the signature $\sigma_{j}=\left(K_{1, i}\right)^{1 / \alpha_{i}+t_{\mathrm{GID}} \beta_{i}}$. Because $G I D, K_{3, i} \in \mathbb{Z}_{p}^{*}$, hence $\left(K_{3, i}, \sigma_{j}\right)$ is a valid signature on message GID in the B-B signature scheme. Because GID $\in\left\{\mathrm{GID}_{1}, \cdots, \mathrm{GID}_{m}\right\}$, it means $\mathbb{B}$ never queried the signature of GID before, and the advantage of $\mathbb{B}$ to break the $B-B$ scheme is equal with the advantage of the adversary $A$ to break our scheme, which is $\varepsilon$. 
TABLE 5: Computational cost comparison.

\begin{tabular}{lcccc}
\hline Scheme & {$[11]$} & {$[13]$} & {$[14]$} & Our scheme \\
\hline Key generation & $3 N_{u} E$ & $5 N_{u} E$ & $\left(4 N_{u}+4\right) E$ & $3 N_{u} E$ \\
Encryption & $\left(N_{u}+5 N_{e}+1\right) E+1 E_{T}+N_{u} P_{e}$ & $\left(2 N_{e}+1\right) E_{T}+6 N_{e} E$ & $\left(5 N_{e}+2\right) E+1 E_{T}$ & $\left(N_{u}+7 N_{e}+1\right) E+1 E_{T}+N_{u} P_{e}$ \\
Outdecryption & $\mathrm{N}_{d} E_{T}+3 N_{d} P_{e}$ & - & $\mathrm{N}_{d} E_{T}+\left(3 N_{d}+1\right) P_{e}$ & $3 N_{d} E_{T}+3 N_{d} P_{e}$ \\
User decryption & $1 E_{T}$ & $4 N_{d} E_{T}+3 N_{d} P_{e}$ & $3 E_{T}$ & $1 E_{T}$ \\
\hline
\end{tabular}

According to the Boneh-Boyen signature scheme, we can also get the following lemma.

Lemma 2. If the q-SDH assumption holds in the group $\mathbb{G}$, the full signature scheme of Boneh and Boyen is strong existential forgery secure against adaptive chosen message attacks.

Theorem 4. If the q-SDH assumption holds in the group $\mathbb{G}$, our scheme achieves fully user traceability.

Proof. It follows directly from the above Lemma 1 and Lemma 2.

\section{Performance Analysis}

The notations used in our performance analysis are summarized in Table 3.

The comparison of storage cost and computational cost between our scheme and some other ABE schemes is illustrated in Tables 4 and 5separately. Notice that all results do not contain the costs of the symmetric cryptography including hash operations.

From Table 4, we can see that the decryption key lengths of scheme [11] and ours are related to the number of attributes used in decryption as both scheme outsource the most decryption work to the cloud server, while the decryption key length of scheme [14] is related to the number of attributes in user attribute sets. Speaking of the length of the ciphertext, of all four schemes are associated with the row number of the encryption LSSS access matrix.

As we can see from Table 4, scheme [13] needs $5 N_{u}$ exponentiations in group $\mathbb{G}$ to generate the user decryption key. It needs $2 N_{e}+1$ exponentiations in group $\mathbb{G}_{T}$ and $6 N_{e}$ exponentiations in group $\mathbb{G}$ in the encryption phase. Specially, 4 $N_{d}$ exponentiations in group $\mathbb{G}_{T}$ and $3 N_{d}$ pairings are costed by a user who needs to decrypt in scheme [13], which is too heavy for resource-limited IoT devices. Scheme [11] is an ABE scheme with outsourced decryption which needs $3 N_{u}$ exponentiations in group $\mathbb{G}$ in the key generation phase. It requires $\mathrm{N}_{u}+5 N_{e}+1$ exponentiations in group $\mathbb{G}$, one exponentiation in group $\mathbb{G}_{T}$, and $\mathrm{N}_{u}$ pairings to encrypt. As the most pairing operations are done by the cloud server, users only cost one exponentiation in group $\mathbb{G}_{T}$ to decrypt in [11].

Li et al. proposed a traceable ABE scheme which needs $4 \mathrm{~N}_{u}+4$ exponentiations in group $\mathbb{G}$ to generate the private key [14]. In encryption phase, users spends $5 N_{e}+2$ exponentiations in group $\mathbb{G}_{T}$ and one exponentiation in group $\mathbb{G}$, while the cloud server performs $\mathrm{N}_{d}$ exponentiations in group $\mathbb{G}_{T}$ as well as $3 N_{\mathrm{d}}+1$ pairings to predecrypt in [14]. As a result, users only cost three exponentiations in group $\mathbb{G}_{T}$ to fully decrypt.

Our scheme needs $3 N_{u}$ exponentiations in group $\mathbb{G}$ in the key generation phase. To achieve fully policy hidden which is deeply valuable in some healthy data application, our scheme requires $\mathrm{N}_{u}+7 N_{e}+1$ exponentiations in group $\mathbb{G}$, one exponentiation in group $\mathbb{G}_{T}$, and $\mathrm{N}_{u}$ pairings to encrypt. Meanwhile, our scheme realizes verifiable outsourced decryption. Our scheme outsources $3 N_{d}$ exponentiations in group $\mathbb{G}_{T}$ and $3 N_{d}$ pairings to the cloud server. Thus, IoT devices in our scheme only require one exponentiation in group $\mathbb{G}_{T}$ to decrypt, which dramaticlly reduces the computational overhead of resource-limited devices.

Figure 7 illustrates the time overhead of decryption. The simulation is performed in a Ubuntu 16.4 desktop system with 3.0-GHz Intel Core (TM) i5-7400 CPU and 2-GB RAM, and all experiments are done by using the Charm (version 0.50) [37], a rapid prototyping framework for cryptographic schemes based with Python.

Compared with the outsourced multiauthority $\mathrm{ABE}$ scheme [11] with no traceability, our traceable MAABE scheme is with little extra computational cost. However, the user decryption cost of [11] and our scheme is same owing to the outsourced decryption. While comparing with the traceable single-authority ABE scheme [14], our multiauthority scheme can handle more attributes and is more suitable for a large number of devices of IoT systems. In addition, another traceable MAABE [13] is not applicable for resource-limited IoT devices due to its heavy decryption cost.

More importantly, our scheme costs barely one hash operation to achieve the verification of decryption results. About another practical function is achieved by our scheme, traceability, and the cost of our scheme is $\mathrm{N}_{a}(2 E+H+3 P)$. Although it looks like that this result is linear to the size of the attribute universe set, the real computational cost of this algorithm for each AA is linear to the size of its own attribute set as we assumed that attribute sets controlled by different attribute authorities are disjoint in our scheme.

\section{Conclusion}

In this paper, we propose a multiauthority $\mathrm{ABE}$ scheme supporting verifiable outsourced decryption and white-box traceability. Our scheme outsources most decryption works to the honest-but-curious resource-rich cloud server; thus, our scheme meets the special needs of resource-limited IoT devices. Moreover, our scheme protects the privacy of both the encryptor and the decryptor by the fully hiding policy technology. At the same time, another issue influences the application of $\mathrm{ABE}$ - the key leakage problem-which is 
solved by the user traceability algorithm. In a word, our scheme realizes several practical functions while achieving replayable chosen-ciphertext attack security.

In the future, we plan to improve the scheme with fixed key size and ciphertext size to further reduce equipment overheads. Moreover, we can also consider how to solve another difficulty of the practical application of the ABE-attribute revocation and user revocation. How to dynamically withdraw attributes or users without affecting other authorized users is the focus of our future works.

\section{Data Availability}

All data used to support the findings of this study are available from the corresponding author upon request.

\section{Conflicts of Interest}

The authors declare that they have no conflicts of interest.

\section{Acknowledgments}

This work was supported in part by the National Key Research and Development Program of China under Grant 2019YFB2102600; in part by the National Natural Science Foundation of China under Grants 62072065, 61832012, 61672321, 61771289, and 61373027; in part by the Fundamental Research Funds for the Central Universities under Grant 2019CDQYRJ006; in part by the Chongqing Research Program of Basic Research and Frontier Technology under Grant cstc2018jcyjAX0334; in part by the Key Project of Technology Innovation and Application Development of Chongqing under Grant CSTC2019jscx-mbdx0151; and in part by the Overseas Returnees Innovation and Entrepreneurship Support Program of Chongqing under Grants cx2018015 and cx2020004.

\section{References}

[1] A. Shamir, "Identity-based cryptosystems and signature schemes," in Workshop on the theory and application of cryptographic techniques, pp. 47-53, Springer, 1984.

[2] D. Boneh and M. Franklin, "Identity-based encryption from the weil pairing," in Annual international cryptology conference, pp. 213-229, Springer, 2001.

[3] A. Sahai and B. Waters, "Fuzzy identity-based encryption," in Annual International Conference on the Theory and Applications of Cryptographic Techniques, pp. 457-473, Springer, 2005.

[4] B. Waters, "Ciphertext-policy attribute-based encryption: an expressive, efficient, and provably secure realization," in International Workshop on Public Key Cryptography, pp. 53-70, Springer, 2011.

[5] S. Pattar, R. Buyya, K. R. Venugopal, S. S. Iyengar, and L. M. Patnaik, "Searching for the iot resources: fundamentals, requirements, comprehensive review, and future directions," IEEE Communications Surveys \& Tutorials, vol. 20, no. 3, pp. 2101-2132, 2018.
[6] M. Chase, "Multi-authority attribute based encryption," in Theory of Cryptography Conference, pp. 515-534, Springer, 2007.

[7] M. Chase and S. S. M. Chow, "Improving privacy and security in multi-authority attribute-based encryption," in Proceedings of the 16th ACM conference on Computer and communications security - CCS '09, pp. 121-130, Chicago Illinois USA, 2009.

[8] A. Lewko and B. Waters, "Decentralizing attribute-based encryption," in Annual international conference on the theory and applications of cryptographic techniques, pp. 568-588, Springer, Tallinn, Estonia, 2011.

[9] M. Green, S. Hohenberger, and B. Waters, "Outsourcing the decryption of abe ciphertexts," in Proc. 20th USENIX Security Symposium, USENIX Association, vol. 2011, pp. 1-16, San Francisco, CA, 2011.

[10] J. Li, F. Sha, Y. Zhang, X. Huang, and J. Shen, "Verifiable outsourced decryption of attribute-based encryption with constant ciphertext length," Security and Communication Networks, vol. 2017, Article ID 3596205, 11 pages, 2017.

[11] S. Belguith, N. Kaaniche, M. Laurent, A. Jemai, and R. Attia, "Phoabe: securely outsourcing multi-authority attribute based encryption with policy hidden for cloud assisted iot," Computer Networks, vol. 133, pp. 141-156, 2018.

[12] Z. Liu, S. Duan, P. Zhou, and B. Wang, "Traceable-then-revocable ciphertext-policy attribute-based encryption scheme," Future Generation Computer Systems, vol. 93, pp. 903-913, 2019.

[13] K. Zhang, H. Li, J. Ma, and X. Liu, "Efficient large-universe multi-authority ciphertext-policy attribute-based encryption with white-box traceability," Science China Information Sciences, vol. 61, no. 3, article 032102, 2018.

[14] Q. Li, H. Zhu, Z. Ying, and T. Zhang, "Traceable ciphertextpolicy attribute-based encryption with verifiable outsourced decryption in eHealth cloud," Wireless Communications and Mobile Computing, vol. 2018, Article ID 1701675, 12 pages, 2018.

[15] H. Zhong, W. Zhu, Y. Xu, and J. Cui, "Multi-authority attribute-based encryption access control scheme with policy hidden for cloud storage," Soft Computing, vol. 22, no. 1, pp. 243-251, 2018.

[16] S. Belguith, N. Kaaniche, A. Jemai, M. Laurent, and R. Attia, "Pabac: a privacy preserving attribute based framework for fine grained access control in clouds," in 13th IEEE International Conference on Security and Cryptography(Secrypt), pp. 133-146, Portugal, 2016.

[17] Z. Cai, Z. He, X. Guan, and Y. Li, "Collective data-sanitization for preventing sensitive information inference attacks in social networks," IEEE Transactions on Dependable and Secure Computing, vol. 15, no. 4, pp. 577-590, 2018.

[18] V. Božović, D. Socek, R. Steinwandt, and V. I. Villányi, "Multiauthority attribute-based encryption with honest-but-curious central authority," International Journal of Computer Mathematics, vol. 89, no. 3, pp. 268-283, 2012.

[19] Z. Cai and X. Zheng, "A private and efficient mechanism for data uploading in smart cyber-physical systems," IEEE Transactions on Network Science and Engineering, vol. 7, no. 2, pp. 766-775, 2020.

[20] C. Hu, N. Zhang, H. Li, X. Cheng, and X. Liao, "Body area network security: a fuzzy attribute-based signcryption scheme," IEEE Journal on Selected Areas in Communications, vol. 31, no. 9, pp. 37-46, 2013. 
[21] X. Zheng, Z. Cai, J. Yu, C. Wang, and Y. Li, "Follow but no track: privacy preserved profile publishing in cyber-physical social systems," IEEE Internet of Things Journal, vol. 4, no. 6, pp. 1868-1878, 2017.

[22] Y. Pu, C. Hu, S. Deng, and A. Alrawais, " $R^{2} P E D S$ : a recoverable and revocable privacy-preserving edge data sharing scheme," IEEE Internet of Things Journal, vol. 7, no. 9, pp. 8077-8089, 2020.

[23] Z. Cai and Z. He, "Trading private range counting over big iot data," in 2019 IEEE 39th International Conference on Distributed Computing Systems (ICDCS), pp. 144-153, Dallas, TX, USA, July 2019.

[24] X. Xu, J. Zhou, X. Wang, and Y. Zhang, "Multi-authority proxy re-encryption based on cpabe for cloud storage systems," Journal of Systems Engineering and Electronics, vol. 27, no. 1, pp. 211-223, 2016.

[25] Y. Yang, H. Zhu, H. Lu, J. Weng, Y. Zhang, and K.-K. R. Choo, "Cloud based data sharing with fine-grained proxy re-encryption," Pervasive and Mobile Computing, vol. 28, pp. 122-134, 2016.

[26] X. Zheng, Z. Cai, and Y. Li, "Data linkage in smart internet of things systems: a consideration from a privacy perspective," IEEE Communications Magazine, vol. 56, no. 9, pp. 55-61, 2018.

[27] J. Lai, R. H. Deng, C. Guan, and J. Weng, “Attribute-based encryption with verifiable outsourced decryption," IEEE Transactions on Information Forensics and Security, vol. 8, no. 8, pp. 1343-1354, 2013.

[28] N. Deng, S. Deng, C. Hu, and K. Lei, "An efficient revocable attribute-based signcryption scheme with outsourced designcryption in cloud computing," in International Conference on Wireless Algorithms, Systems, and Applications, pp. 84-97, Springer, 2019.

[29] T. Nishide, K. Yoneyama, and K. Ohta, “Attribute-based encryption with partially hidden encryptor-specified access structures," in International conference on applied cryptography and network security, pp. 111-129, Springer, 2008.

[30] M. J. Hinek, S. Jiang, R. S. Naini, and S. F. Shahandashti, "Attribute-based encryption without key cloning," International Journal of Applied Cryptography, vol. 2, no. 3, pp. 250270, 2012.

[31] D. Boneh and X. Boyen, "Short signatures without random oracles," in International conference on the theory and applications of cryptographic techniques, pp. 56-73, Springer, 2004.

[32] Zhen Liu, Zhenfu Cao, and D. S. Wong, "White-box traceable ciphertext-policy attribute-based encryption supporting any monotone access structures," IEEE Transactions on Information Forensics and Security, vol. 8, no. 1, pp. 76-88, 2013.

[33] Z. Liu, Z. Cao, and D. S. Wong, "Traceable cp-abe: how to trace decryption devices found in the wild," IEEE Transactions on Information Forensics and Security, vol. 10, no. 1, pp. 55-68, 2015.

[34] G. Yu, Y. Wang, Z. Cao, J. Lin, and X. Wang, “Traceable and undeniable ciphertext-policy attribute-based encryption for cloud storage service," International Journal of Distributed Sensor Networks, vol. 15, no. 4, 2019.

[35] H. Qiao, J. Ren, Z. Wang, H. Ba, and H. Zhou, “Compulsory traceable ciphertext-policy attribute-based encryption against privilege abuse in fog computing," Future Generation Computer Systems, vol. 88, pp. 107-116, 2018.
[36] R. Canetti, H. Krawczyk, and J. B. Nielsen, "Relaxing chosenciphertext security," in Annual International Cryptology Conference, pp. 565-582, Springer, 2003.

[37] J. A. Akinyele, C. Garman, I. Miers et al., "Charm: a framework for rapidly prototyping cryptosystems," Journal of Cryptographic Engineering, vol. 3, no. 2, pp. 111-128, 2013. 